\title{
Optional Disclosure and Observational Learning
}

\author{
Diefeng Peng, Yulei Rao, Xianming Sun, and Erte Xiao*
}

\begin{abstract}
:
Observational learning theories often assume that people's actions can be observed. However, in many naturally-occurring environments, individuals can choose whether to disclose their behavior to others. We provide theoretical analysis of observational learning under optional disclosure conditions. We further examine empirically how individuals decide whether to reveal decisions. Although we find evidence for other-regarding disclosure behavior, our findings highlight the importance of providing public information about how the disclosure behavior affects others.
\end{abstract}

Keywords: Observational learning; Information cascade; Optional disclosure; Other-regarding preferences

JEL Codes: C91, D82, D83, D64, D91

\footnotetext{
* Corresponding author: Xiao (erte.xiao@monash.edu) is from Department of Economics, Monash University. Peng (stephen@csu.edu.cn) and Rao (yuleirao@sina.com) are from School of Business, Central South University; Sun (Xianming.Sun@zuel.edu.cn) is from School of Finance, Zhongnan University of Economics and Law. We are grateful to Daniel Houser, Jianxin Wang, Tracy Xiao Liu, Sen Geng, and to seminar participants at the XMU Experimental 2014, International Economic Science Association 2015 and North-American ESA conference 2017 for helpful comments and suggestions. We acknowledge the financial support from the National Natural Science Foundation of China (Grant Numbers: 71673306, 11571373).
} 


\section{Introduction}

Individual decision-making is often influenced by one's observations of what others have done. In this way, observational learning helps to provide people with the superior information to make better decisions. Indeed, welfare may improve when decisionmakers learn from others rather than merely relying on their own private information. This is supported by both theoretical and empirical evidences (Bikhchandani et al., 1998; Hung \& Plott, 2001; Weizsäcker, 2010; Lee et al., 2015).

On the other hand, observational learning can lead to information cascades when people abandon their own information and make decisions based only on inferences from what others have done (Bikhchandani et al., 1992; Anderson \& Holt, 1997; Hung \& Plott, 2001; Nöth \& Weber, 2003; Goeree et al., 2007). When cascades form, public information stops accumulating and learning is blocked. Also,cascades can be incorrect (Bikhchandani et al., 1992). For example, online markets often provide the statistics of monthly sales of products. Such information, based on previous consumers' purchasing behavior, may signal the quality of the products and therefore improve consumers' purchasing decisions. However, it can also mislead consumers when the initial purchasing behavior does not reflect the true quality of the product ${ }^{1}$.

Critically, the current theory and experimental literature on observational learning and information cascade assumes that individuals' choices are automatically revealed to the public (Anderson \& Holt, 1997; Bikhchandani et al., 1998). In many naturallyoccurring environments, however, individuals have the option to decide whether to disclose their behavior to others (i.e., endogenous information sharing). Take the above example of online shopping. While many websites (e.g., tmall.com in China) allow consumers to sort products based on "best sellers," some other websites (e.g., Suning.com in China) introduce "most reviewed" as the main sorting algorithm. These two sorting algorithms imply different disclosure mechanisms. Under the "best sellers" algorithm, consumers' purchasing decisions are automatically recorded and revealed. In contrast, under the "most reviewed" algorithm, customers' purchasing decisions are counted only when they choose to leave feedback and disclose their purchasing decisions. Little is known regarding the economic implications of allowing decision-

\footnotetext{
${ }^{1}$ For example, authors may manipulate the best seller list by privately purchasing a large quantity of their own books (Bikhchandani et al., 1998). A bank run is another example on the dark side of cascades (Diamond \& Dybvig, 1983).
} 
makers to choose whether to reveal their choices to the public. Further, how does one decide whether to reveal his/her decisions to others? How will disclosure behavior affect observational learning?

We tackle these questions in this paper. We first extend the existing models of observational learning by considering endogenous information-sharing with disclosure options. In the classical models of information cascades (e.g., Bikhchandani et al. 1992), a sequence of individuals makes payoff-relevant guesses between two exclusive alternatives in an exogenously determined order. The guesses of all the predecessors are automatically made public and fully observed by others. In this setup, individuals can make better decisions by inferring information from the actions of predecessors than by relying on their private information alone. However, information cascades arise after very few periods and consequently lead to behavior convergence and bring information updating to a standstill. Therefore, the incremental decision efficiency that individuals acquire from observational learning is limited due to the low quality of public information. In our set up, we allow agents to choose whether they want their guesses to be observed by others (in particular, the subsequent decision-makers). We thereby relax the assumption of automatic publicity of predecessors' guesses.

Theoretically, we identify two opposing effects of endogenous information sharing. The first is a pure information effect due to reduced information available to the market when some guesses are hidden. The missing information effect is determined by the likelihood of revealing when one's guess is consistent with one's own private signal (denoted as $k$ in the model). The second effect, which we call selectiveness, improves the quality of available information. This effect is determined by the difference in the probability of revealing when one's guess is consistent with her private signal and when they are not (denoted as $\delta$ in the model). The higher the values of $k$ and $\delta$, the more likely the selectiveness effect dominates the negative missing information effect and thereby increases the decision efficiency (i.e., the total number of correct guesses).

If individuals are purely egocentric, we would expect people to disclose their guesses in a random pattern independent of the nature of the guesses (i.e., $k=1 / 2$ and $\delta=0$, or unselective disclosure). In this case, optional disclosure simply reduces the available information. Compared with the traditional automatic information sharing condition ( $k=1$ and $\delta=0)$, endogenous information sharing leads to lower decision 
efficiency. On the other hand, considerable research has shown that individuals have other-regarding preferences (Fehr \& Schmidt, 1999; Charness \& Rabin, 2002; Andreoni \& Miller, 2002). We hypothesize that other-regarding preferences play a role in the disclosure decisions, and that pro-social decision-makers will adopt disclosure strategies that help subsequent players make better decisions. Thus, we predict the values of $k$ and $\delta$ will be higher than those under the assumption of selfishness: $k>1 / 2$ and $\delta>0$. We conduct a series of controlled laboratory experiments to test this prediction, and shed light on the decision-making involved in information sharing.

The baseline treatment is built on the information cascade experiment first conducted by Anderson \& Holt (1997). Each participant is told that there are two possible color combinations of balls in an unmarked urn. Each participant draws from the urn (after which the balls are replaced), and is then asked, sequentially, to guess which color combination they have drawn. Each participant's guess is then automatically shown to the other participants.

We compare the outcome in this baseline with that in a new treatment: the optional disclosure treatment (OD), in which subjects also decide whether to reveal their guess to the subsequent players when making their own guess. We examine, without any external interventions, how often participants reveal their guesses in various scenarios, and, in particular, the values of $k$ and $\delta$. We find that, on average, participants reveal their guess $76.8 \%$ of time when their guess is consistent with their private information. The disclosure frequency is about $30 \%$ higher than when the guesses are inconsistent with the private signals. These results support the hypothesis of otherregarding preference in the disclosure decisions.

Interestingly, we find that the endogenous information sharing condition hinders observational learning. Compared with the baseline, in the OD treatment, decisionmakers are significantly more likely to guess based on their own private information and disregard the public information regardless of whether the public information produces correct guesses. One possible reason for this reduced observational learning is that the missing information under the optional disclosure makes it more difficult for decisionmakers to make use of the public information, even though the signal-consistent guesses are more likely to be disclosed than the inconsistent ones. That is, the cost of missing information dominates the benefit of selective disclosure. We report data from another 
treatment where the computer randomly decides whether to publicize each subject's guess to the other players (CD). The distribution of the disclosure probability is kept the same as in the OD treatment. Thus, any difference in the learning outcomes between the $\mathrm{CD}$ and the OD treatment reveals the significance of the selectiveness effect. We find similar learning outcomes in the $\mathrm{CD}$ and $\mathrm{OD}$ treatments, which suggests that the selectiveness effect is minor in the OD treatment.

Combined with the theoretical findings, one explanation for the dominant missing information effect is that the values of $k$ and $\delta$ are not high enough. There are two possible reasons for the relative low values of $k$ and $\delta$. One is that the role of other-regarding preferences on disclosure choice is limited. Another possibility is that many agents are motivated to help others, but do not know how to do it. While the first explanation suggests that additional incentives should be provided to align the interests of the agents, the second explanation implies that simply providing public information as to the most socially efficient information-sharing behavior can promote efficient observational learning. To shed light on these two possible reasons, we conducted a second study. The main treatment of the second study is the informed optional disclosure (IOD) treatment. The only difference between the IOD and the OD treatments is that participants were informed that in a previous survey a large proportion of participants believed that disclosing only private-signal-consistent guesses could help the subsequent players. If some agents intended to help others, but adopted incorrect strategies in the OD treatment, we should observe the values of $k$ and $\delta$ increase in the IOD treatment. We find this is indeed the case. Moreover, the higher $k$ and $\delta$ values improve observational learning.

Like the first study, we also compare IOD with another treatment (CD_IOD) where the disclosure decisions were randomly determined by the computer, but the distribution was kept the same as the IOD. We find significant treatment differences in the observational learning. Thus, we show that it is the improved selectiveness effect that facilitates observational learning in the IOD treatment. Our findings highlight the importance of introducing additional guidelines on how disclosure decisions may affect others' guesses under the optional disclosure condition.

The rest of the paper is organized as follows. Section 2 reviews previous related literature. section 3 provides theoretical analysis and derive predictions of the disclosure 
behavior of other-regarding agents. In section 4, we design an experiment based on Anderson \& Holt (1997) to provide the first empirical evidence on how agents choose whether to disclose their guesses. Section 5 reports the results. Sections 6 and 7 report findings from three additional treatments that add to our understanding of disclosure decisions and the impact of optional disclosure on observational learning outcomes. Section 8 concludes.

\section{Related Literature}

A large body of literature has studied the interaction between private belief and public information through observational learning. Banerjee (1992) and Bikhchandani et al. (1992) (henceforth, BHK) constructed a theory of information cascades. They found that people rationally choose to follow the established pattern, rather than their own signals, when the public information overweighs their private information. Anderson \& Holt (1997) (henceforth, AH) conducted the first laboratory study of information cascade and found experimental evidence for the rational conformity behavior. They showed that observational learning is economically rational, but has the potential risk of erroneous cascades.

Others have extended and modified AH's experiment from various perspectives. Hung \& Plott (2001) compared the impacts of various institutions on the efficiency of sequential decisions. Nöth \& Weber (2003) introduced different signal qualities to identify subjects' overconfidence (which has been argued to reduce the likelihood of cascades). Goeree et al. (2007) varied the length of decision sequence and signal informativeness. Ziegelmeyer et al. (2010) analyzed the fragility of information cascades by eliciting beliefs about signals and states of the world. Fahr \& Irlenbusch (2011) examined the difference in learning behavior between small groups and individuals. Experiments have also been conducted to understand the herding behavior in financial markets (Cipriani \& Guarino, 2005; Drehmann et al., 2005; Alevy et al., 2007).

Some recent papers investigated endogenous information acquisition where decision makers have an option to choose whether to obtain information from others (Kübler \& Weizsäcker, 2004; Kultti \& Miettinen, 2006, 2007; Celen et al., 2010; 
Acemoglu et al., 2011; Çelen \& Hyndman, 2012; Smith \& Sorensen, 2008; Goeree \& Yariv, 2015; Lobel \& Sadler, 2015; Song, 2016). Our paper complements this literature by examining endogenous information sharing. To our knowledge, this is the first paper that theoretically and empirically investigates observational learning under the condition of optional disclosure. By examining optional disclosure, we extend the current social preference literature to the research of observational learning and information cascades.

Considerable research has shown that individuals incorporate others' payoffs into their utility function. People are even willing to incur costs to promote others' welfare (Andreoni \& Miller, 2002; Fehr \& Fischbacher, 2003; Cooper \& Kagel, 2015). Yet, little attention has been paid to the role of pro-social preferences in observational learning with only a few exceptions. Ali \& Kartik (2012) developed a simple model of observational learning under that assumption of collective preference. Davis (2017) examined their prediction in a lab experiment and found that payoff interdependence had no influence on cascade behavior. Ash \& Van Parys (2015) found that information cascades were more likely when subjects were conscious of their membership in certain groups. March \& Ziegelmeyer (2016) provided direct evidences of the altruistic explanation for decision makers' relative overemphasis on private information. In all these studies, to help the subsequent players one had to respond to his/her own private information even if he/she would be better off by herding. As elaborate below, by introducing optional disclosure, we allow decision makers to use the disclosure decisions to signal the private information and still make a profit-maximizing guess for themselves. Our findings offer a new model on the role of other-regarding preference in observational learning.

\section{Theoretical analysis}

Let the random nature be characterized by $(\Omega, \mathbb{P})$, where $\omega \in \Omega=\{1,-1\}$ denotes the state of the nature and $\mathbb{P}$ is the common prior probability measure on $\Omega$. Following Bikhchandani et al. (1992), we set $\mathbb{P}(\omega=1)=\mathbb{P}(\omega=-1)=0.5$. A finite set of agents, $T$, take part in a guessing game where agent $t \in\{1, \ldots, T\}$ has to make a guess $a_{t} \in \mathcal{A}=\{1,-1\}$ at the position $t=1, \ldots, T$. Their guesses are automatically 
revealed to the public, which is called previous-action-observable (PAO) assumption. The ordering of the positions is exogenously determined and is known to all agents. Once the game starts, the nature state $\omega$ simultaneously fixes the correct answer, which is unknown to all the agents until the end of this game. An agent will get $\$ 1$ if her guess is correct, $a_{t}=\omega$, otherwise she gets $\$ 0$. Before making her guess, she receives a private signal $s_{t}$ which matches the true state with the probability of $q$. That is,

$$
q=\mathbb{P}\left(s_{t}=1 \mid \omega=1\right)=\mathbb{P}\left(s_{t}=-1 \mid \omega=-1\right),
$$

where $q \in(1 / 2,1)$.

In the PAO sequential learning case, an agent $t$ observes the full history of previous guesses $H_{t}=\left\{a_{i}\right\}_{i=1}^{t-1}$ (Bikhchandani et al., 1992). The Bayesian Nash equilibrium is

$$
a_{t}= \begin{cases}s_{t}, & \left|h_{t}\right| \leq 1, \\ \frac{h_{t}}{\left|h_{t}\right|}, & \left|h_{t}\right|>1,\end{cases}
$$

where $h_{t}=\log _{\frac{q}{1-q}}\left[\frac{\mathbb{P}\left(H_{t} \mid \omega=1\right)}{\mathbb{P}\left(H_{t} \mid \omega=-1\right)}\right]$ is the public belief after history $H_{t}$ (Zhang, 2015). Equation (2) indicates that the public belief in the PAO setting evolves in the following way

$$
h_{t+1}= \begin{cases}h_{t}+s_{t}, & \left|h_{t}\right| \leq 1, \\ h_{t}, & \left|h_{t}\right|>1 .\end{cases}
$$

Equation (3) means once $\left|h_{t}\right|>1\left(h_{t}=-2\right.$ or $\left.h_{t}=2\right)$, agent $t$ discards her private information and follows the crowd and so do agents $t+1, t+2$, and so on. In this setting, individuals maximize their profit by learning from their predecessors' guesses that are automatically disclosed. However, such observational learning prevents the public belief from being updated.

We now explore what might happen if agents could decide whether to disclose their guesses. Since an agent's payoffs do not depend on what later agents do, if she is a self-interest maximizer, she may not take into account the impact of her guess on the subsequent players. The probability for this agent to reveal her guess should be independent of the nature of the guess. Optional disclosure will not improve collective decision-making, but merely reduce the observable information, making it even inferior to the PAO setting (see Proposition 1). 
However, if an agent has other-regarding preference, she may be motivated to use a certain disclosure strategy to help subsequent players make better guesses. Below, we derive the conditions for the disclosure strategies to be socially beneficial. There may be various disclosure strategies to help others. Here, we consider the case when the choice of disclosure is selective, in that it is correlated with the consistency between the guess and the private signal. This seems to be the easiest and most straightforward approach for signaling one's private information.

Let us first classify guesses based on their consistency with the private signal.

\section{Definition 1.}

(i) A guess is called a signal-consistent guess if it is consistent with one's own private signal.

(ii) A guess is called a signal-inconsistent guess if it is inconsistent with one's private signal.

To formulate disclosure strategies, we denote $d_{t}=1$ if an agent $t$ chooses to disclose her guess; otherwise, $d_{t}=0$. Let $k(0 \leq k \leq 1)$ denote the probability that an agent discloses a signal-consistent guess, and $k-\delta(0 \leq \delta \leq k)$ denote the probability that an agent discloses a signal-inconsistent guess. We define the selectiveness of disclosure as the difference of disclosure probability between two types of guesses measured by $\delta$, a key parameter in formulating disclosure strategies, i.e.,

$$
\mathbb{P}\left(d_{t}=1 \mid a_{t} s_{t}=1\right)=k \quad \text { and } \quad \mathbb{P}\left(d_{t}=1 \mid a_{t} s_{t}=-1\right)=k-\delta .
$$

Based on the above parametric setting, we characterize disclosure behavior as follows:

\section{Definition 2. ,}

Optional disclosure may result in one of the following four types ${ }^{2}$ :

(i) full disclosure if $\delta=0$ and $k=1$.

\footnotetext{
${ }^{2}$ Our analysis focuses on $\delta \geq 0$. The reason is that our goal is to identify disclosure strategies that can help subsequent players. If $\delta<0$, agents are more likely to disclose a signal inconsistent guess than a consistent one. It is easy to find out that such a strategy cannot be welfare improving. We can see this by using an extreme example where subjects always disclose signal inconsistent guesses, and never disclose signal consistent guesses $(k=0$ and $\delta=-1)$. Since the first agent has only her private signal, a rational guess would be a signal consistent guess. Thus, the first agent would not disclose her guess. This means, the second player will also have her own private signal and subsequently make a signal consistent guess and will not reveal the guess. The same is true for all the other players. Thus, in this case, social learning will never happen and the outcome is equivalent to the independent individual decision-making.
} 
(ii) selective (partial) disclosure if $0<\delta \leq k$ and $0<k \leq 1$.

(iii) unselective (partial) disclosure if $\delta=0$ and $0<k<1$.

(iv) no disclosure if $\delta=0$ and $k=0$.

"Full disclosure" is equivalent to the PAO setting in the BHK model as discussed above. "No disclosure" means no social learning and each participant makes her guess only based on her private information. Our analysis below therefore focuses on the two partial disclosure types.

When $\delta=k=1$, agents always disclose the signal-consistent guesses and never disclose the signal-inconsistent ones. In this case, agents are perfectly informative and selective in disclosing their guesses. If this disclosure strategy is common knowledge and adopted by every agent, then each observable guess reveals its underlying private signal and each hidden guess means the private signal contradicts with the observed public information. In this way, agents' private information is fully revealed to the public. Hence, perfectly informative and selective disclosure is equivalent to what Bikhchandani et al. (1992) called previous-signal observable (PSO) regime, where an incorrect cascade can be switched by a long enough series of opposing signals.

However, empirically it is more likely that $\delta=k=1$ is not satisfied. In this case, compared with full disclosure (the PAO setting), the optional disclosure can have two impacts: a pure information effect and a selectiveness effect. As long as $k<1$, one will have less information available when making his/her guess. We define "pure information effect" as the case when $k<1$ and $\delta=0$. In other words, unselective disclosure, as defined in Definition (iii) will have only the pure information effect. To learn the consequences of optional disclosure, we derive how the pure information effect and the selectiveness effect influence individual's decision-making respectively.

\section{Proposition 1. (Pure information effect) :}

Denote by $k_{1} \in[0,1]$ and $k_{2} \in[0,1]$ two alternative values of the probability that agents disclose a signal consistent guess, such that $k_{1}<k_{2}$. For any $q \in(1 / 2,1)$, if $T \geq 2$, we have for any $t, t \in\{2,3, \ldots, T\}$,

(i) $\mathbb{P}\left(\left|h_{t}\right|>1 \mid k=k_{1}, \delta=0\right)<\mathbb{P}\left(\left|h_{t}\right|>1 \mid k=k_{2}, \delta=0\right)$.

(ii) $\mathbb{P}\left(a_{t}=\omega \mid k=k_{1}, \delta=0\right)<\mathbb{P}\left(a_{t}=\omega \mid k=k_{2}, \delta=0\right)$. 
Proof. See Appendix C.

Proposition 1 indicates that compared with PAO setting, with unselective disclosure $(\delta=0)$, the more signals that are hidden, the less likely public belief will evolve to the point beyond which subsequent agents choose to neglect their private signals. This means that unselectively partial disclosure would delay the starting time of information cascade. Besides, for any agent $t(t>1)$, the chance for her to make a correct guess decreases, as an incompletely displayed history of actions is less informative than a complete one.

When agents are purely egocentric, they are not motivated to use disclosure decisions to help others. For any type of guess, a purely selfish agent is indifferent between revealing and not revealing it: $k=1 / 2, \delta=0$. On the other hand, the pure information effect implies that when an agent adopts an unselective disclosure strategy $(\delta=0)$, the more likely he/she reveals the guess (a high $k$ ), the better off the subsequent players are. Thus, if an agent intends to help other players, he/she should have $k>1 / 2$. Next we show in Proposition 2 that agents can help the subsequent players further with a selective disclosure strategy $(\delta>0)$.

\section{Proposition 2. (Selectiveness effect) :}

For any $q \in(1 / 2,1)$ and $k \in(0,1]$, there must be a finite number $t_{0}\left(t_{0}>0\right)$ to satisfy that

$$
\mathbb{P}\left(a_{t}=\omega \mid 0<\delta \leq k, t>t_{0}\right)>\mathbb{P}\left(a_{t}=\omega \mid \delta=0, t>t_{0}\right) .
$$

Proof. See Appendix C.

Proposition 2 argues that for any selective disclosure strategy with $0<\delta \leq k \leq 1$, there will be a position $t_{0}$ such that, compared with the unselective disclosure $(\delta=0)$ with the same $k$, the selective disclosure can increase the probability of correct guessing made by all the agents after the position $t_{0}$.

\section{[Insert Figure 1 ]}

We conduct simulations to examine in detail how the probability of correct guessing changes on $k$ and $\delta$. The simulation results reveal that the accuracy rate is monotonously increasing in $k$ and $\delta$ (see Appendix B). Figure 1 provides some examples of the simulation results. In each example, we include three benchmarks: PAO condition 
( $k=1$ and $\delta=0)$; the pure egocentric disclosure strategy case $(k=1 / 2$ and $\delta=0)$; and the perfectly informative and selective disclosure strategy case $(k=\delta=1)$. The simulation results provide a few insights as follows.

First, consistent with theoretical predictions, compared with the PAO condition, players can be worse off in the endogenous information sharing condition if each player is egocentric and is indifferent between revealing and not revealing her guess $(k=1 / 2$ and $\delta=0$ ). Second, the probability of correct guessing for agent $t$ after a certain position increases with $k$ and $\delta$. For instance, panel (b) in Figure 1 shows that when $k=1$, starting from the $5^{\text {th }}$ agent, the higher $\delta$ is the more likely an agent is to make a correct guess. Third, as illustrated by panel (d), while the first several agents may be worse off in the endogenous information sharing condition than in the PAO condition due to the missing information $(k<1)$, agents in the later sequence are likely to be better off if selectiveness effect is positive $(\delta>0)$. Moreover, the greater $k$ and $\delta$, the more players (the smaller $t_{0}$ ) can benefit from endogenous information sharing.

In sum, our theoretical analysis points out two opposing effects of endogenous information sharing in observational learning. One is the negative missing information effect and the other is the positive selectiveness effect. We identify two parameters $k$ and $\delta$ that determine the degree of these two effects. The theoretical analysis thus generate two predictions in view of the hypothesis that other-regarding preferences play a role in the disclosure decisions:

Prediction 1. : According to Proposition 1 (the pure information effect), otherregarding players have a higher $k$ than the purely selfish players (i.e., $k>1 / 2$ ).

Prediction 2. : According to Proposition 2 (the selectiveness effect), other-regarding players have a higher $\delta$ than selfish players (i.e., are more likely to reveal the signal consistent guesses than signal inconsistent guesses $(\delta>0))$.

We conducted controlled laboratory experiments to test these two predictions and provide empirical evidence that sheds light on the information sharing behavior. 


\section{Experiment}

\subsection{Experiment Design}

Our baseline treatment was built on the classic information cascade experiment by $\mathrm{AH}$. Each session consisted of 10 rounds. Ten subjects randomly formed a group and remain in the same group for 10 rounds. Subjects were told that there were two urns. Each contained different numbers of red and white balls: either two RED balls and one WHITE ball (called RED urn), or two WHITE balls and one RED ball (called WHITE urn). At the beginning of each round, the computer would randomly select one urn with an equal probability.

In each round, the ten subjects in a group were chosen in a random order, and observed a private draw by the computer from the urn, with replacement. After seeing a private draw, each subject guessed the color combination of the balls. His/her guess was subsequently displayed to all the other nine subjects. That is, starting from the second subject, before submitting his/her guess, each saw both the history of the previous guesses and his/her private draw. After all the ten subjects had finished the guesses, a new round started and the decision sequence was randomly reordered at the beginning. Each round proceeded in the same way.

The only difference between the optional disclosure treatment (OD) and the baseline treatment is that, in addition to making the guess, each subject had to decide whether to reveal his/her guess to all the other nine subjects in his/her group. The displayed history of the guesses thus showed only those guesses of subjects who were willing to disclose. If any guess was hidden, it was shown as "not revealed" in the history record. For example, suppose the guesses of the first two subjects were red and white. However, the third subject decided not to reveal his/her guess. In this case, the history record would be shown as "the first player's guess: red urn; the second player's guess: white urn; the third player's guess: not revealed." (see Appendix A for instructions)

\subsection{Experimental Procedure}

The experiments were conducted at the Experiment Center for Behavioral Science at Central South University, using the software z-Tree (Fischbacher, 2007). We ran four sessions for each treatment with 20 subjects in each session. At the beginning of the 
experiment, subjects were randomly divided into two groups of 10 subjects. Each subject played with the same group for 10 rounds $^{3}$. Similar to AH experiment, there was one practice round before the 10 rounds started. Every subject earned a show-up bonus of $15 \mathrm{RMB}$. At the end of the session, one round was randomly selected and each subject was paid $40 \mathrm{RMB}$ if his/her guess was correct in that round, and zero if the guess was wrong.

\section{Results}

We begin with the analysis of disclosure decisions in the OD treatment to test Prediction 1 and Prediction 2. Then we compare the observational learning behavior in the OD and the baseline treatments. In the theoretical analysis, we follow Bikhchandani et al. (1998) and assume rationality in that agents always conform to the public information if and only if the observed imbalance of predecessors' color choices is greater than one. Experimental evidences have shown that people often do not follow the crowd when they should, due to the fact that they give too much weight to their private information (Weizsäcker, 2010). As a result, the actual accuracy rate of the guesses largely depends on how players learn from the public signals. It is thus important to examine the treatment effect on observational learning.

\subsection{Disclosure decisions in the OD treatment}

Following the theoretical analysis, we calculate the average disclosing rate for each type of guess ${ }^{4}$. In the OD treatment, on average, about $76.8 \%$ of the signal-consistent guesses are revealed, which is significantly higher than $50 \%$ (sign test, $p<0.01)^{5}$. In contrast, the disclosure rate of signal-inconsistent guesses is significantly lower $(48.7 \%$ vs. $76.8 \%$, Mann-Whitney test, $p<0.01$ ).

\footnotetext{
${ }^{3}$ In one session of the OD treatment, one computer crashed in the ninth round. Thus, we obtained data only from the first eight rounds for this session.

${ }^{4}$ In the theoretical analysis, we assume agents rationally make a signal inconsistent guess only when it conforms to the public information as this is consistent with profit optimization. In the OD experiment, however, we observe in 44 cases out of 148 signal-inconsistent guesses, participants' guesses are irrational in that they are also inconsistent with the public information, with $61.4 \%$ of them being disclosed. To allow the testing of the theory predictions, we focus only on the signal-inconsistent guesses that satisfy the assumption of rationality.

${ }^{5}$ Unless noted otherwise, in all the non-parametric tests reported below, we calculate the average proportion for each participant over the 10 rounds and treat each individual average as an independent observation.
} 
We also conducted a random effect probit regression analysis of the disclosure decision using only the data of two types of guesses in OD treatment (see Table 1). The independent variables include a dummy for whether the guess is signal inconsistent, a round variable $(r=1, \ldots, 10)$ and a position variable $(t=1, \ldots, 10)$. Consistent with the result from the non-parametric test, we find the coefficient of the signal-inconsistent guess dummy is significantly negative (Z-test, $p<0.01)^{6}$.

\section{[Insert Table 1]}

We next examine individual types. Table 2 summarizes the data. Majority subjects (60 out of 80) have made both signal consistent and signal inconsistent guesses. First, we note that very few players seem to be indifferent between revealing and not revealing their guesses $(k=0.5$ and $\delta=0)$. This suggests that participants do put thought into their disclosure decisions. More importantly, out of the 60 subjects, 27 subjects' disclosure decisions are consistent with the predictions of other-regarding preference $(k>0.5 ; \delta>0)$. In particular, the disclosure decisions of 12 out of these 27 subjects are perfectly selective and informative in that they disclose only signal consistent guesses and never disclose any signal inconsistent guesses $(\delta=k=1)$. We also observe 15 of the 60 subjects are more likely to disclose the signal-inconsistent guesses than the signal consistent ones $(\delta<0)$. One possible reason for the $\delta<0$ disclosure strategy is the image concern, which is not considered in our current theoretical model. For example, one may think that if her disclosed guess turns out to be wrong, it could harm her image more if she is among the few players who guessed wrong than if she is among the majority who were wrong ${ }^{7}$.

For the 20 subjects who made only signal consistent guesses, we cannot know the selectiveness of their disclosure guesses. As it is always socially beneficial to reveal these guesses, we examine how often they disclosed. We find 12 out of the 20 subjects

\footnotetext{
${ }^{6}$ The significant positive $\beta_{2}$ also suggests that people are more likely to disclose their guesses the later position they are in the sequence. One possible explanation is that people in the later positions are more confident that their guesses will be correct and could help the other players. This would be consistent with the other-regarding preference.

${ }^{7}$ Although in principle one can signal the private information by adopting $\delta<0$ strategy, compared with $\delta>0$ strategy, this is a much less straightforward approach and should be less likely to be considered by those other-regarding players who intend to help the others. It may also lead to a less efficient outcome, as we explained in Footnote 2. We also show later that the proportion of this type does not seem to change much when players are given information what disclosure strategy could help subsequent players. This additional results suggest that the $\delta<0$ type is unlikely to be driven by other-regarding preference.
} 
disclosed more than half of the time (i.e., $k>0.5$ ) which is consistent with the otherregarding preference.

In sum, although the theoretically socially beneficial disclosure strategy (i.e., selective disclosure with $\delta>0$ ) is the most popular one, the overall pattern is quite mixed. In Section 7, we explore further why some players' disclosure strategies are not consistent with the socially beneficial strategy. The mixed patterns of disclosure behavior may also interfere with the positive selectiveness effect and contribute to the outcome of reduced observational learning, as we show next.

\section{[Insert Table 2]}

\subsection{Observational learning in the OD and the baseline treatments}

To compare how subjects use public information in each treatment, we define two types of information using fully informed guesses as a benchmark. Fully informed guesses are the guesses that an expected utility maximizer would make if she had access to the entire sequence of realized signals (Hung \& Plott, 2001). If public information predicts the same color with the fully informed choice, we call it favorable public information. Otherwise, we call it unfavorable public information. We focus on the situations where the observed imbalance of predecessors' color guesses is greater than one. One is more likely to make a correct guess if he/she follows the favorable public information and discards the unfavorable information. The accuracy of the guesses thus largely depends on how players learn from the public information.

Figure 2 reports the frequency of following each type of information by treatments. First, as expected, in both treatments, subjects are much more likely to follow the favorable information than the unfavorable information. Interestingly, although players in the OD treatment are less likely to follow the unfavorable information than those in the baseline (15.4\% vs. 39.3\%, Mann-Whitney test, $p<0.01$ ), we also observe such a decrease in the conformity to the favorable information. The proportion of favorable information being followed in the OD treatment is about $7 \%$ lower than in the baseline treatment, and the difference is marginally significant (95\% vs. 88\%, Mann-Whitney test, $p=0.079)$.

These results suggest that partially disclosed information diminishes observational learning, in that decision-makers become less likely to rely on the public information 
regardless of the nature of the information. Such a negative impact on observational learning can lower the rate of correct guesses compared to the baseline treatment. The reason is that it is more likely for a player to receive favorable information than unfavorable information. Thus, the overall accuracy rate relies more on following favorable public information than abandoning unfavorable information. To check this, we compare the accuracy rate between the two treatments.

\section{[Insert Figure 2]}

In theory, the accuracy rate is defined as the probability that the actual guess is the same as the actual state. However, in our experiment, with 10 random draws, there is a chance of more than $20 \%$ that the signal of majority draws contradicts with the true underlying state. The state of nature is unobservable in the experiment (as in many of the naturally-occurring environments). So with a relatively small sample size, it may be improper to use the actual state as a benchmark to measure accuracy. ${ }^{8}$ Thus, we follow Hung \& Plott (2001) and measure the accuracy rate as the proportion of individual guesses that are the same as the fully informed ones ${ }^{9}$. In addition, the findings from the theoretical analysis show that the treatment effect can appear only later in the sequence (see Figure 1). Thus, we compare here the average accuracy rate of the last five subjects instead of the entire sequence. We find that, consistent with our conjecture, the average accuracy rate of the last five subjects in the OD treatment is lower than that in baseline treatment, but the difference is small and not significant statistically $(76.5 \%$ vs. $78.2 \%$, $t$-test, $p=0.74) .^{10}$

\subsection{Discussion}

The data reported above support Predictions 1 and 2 and are consistent with the hypothesis that other-regarding preference plays a role in the disclosure decision. However, the other-regarding disclosure decisions do not seem to improve the outcome

\footnotetext{
${ }^{8}$ To the best of our knowledge, Weizsäcker (2010) is the only paper to use empirically optimal guess (actual state) as the benchmark. The reason is that he builds his analysis on a large meta set, which we do not have.

${ }^{9}$ For example, suppose we observed 8 RED guesses and 2 WHITE guesses in a certain round where 6 RED balls or 4 WHITE balls were drawn from a WHITE urn. The fully informed guess is RED instead of WHITE. Thus, the accuracy rate for this round is 0.8 instead of 0.2 .

${ }^{10}$ The accuracy difference for the first five subjects between OD treatment and baseline treatment is also quite small and not significant statistically $(68.4 \%$ vs. $71.2 \%, t$-test, $p=0.55)$.
} 
of observational learning. We find that under the optional disclosure condition, players become more likely to discard public information even when they should conform. As a result, the overall accuracy rate does not differ or is even slightly lower than the baseline treatment.

One possible reason for this reduced observational learning is that the missing information from previous guesses makes it more difficult for decision-makers to make use of the public information. That is, the negative impact of missing information dominates the benefit of selective disclosure. To shed light on this possibility, we further conducted a computer disclosure treatment $(\mathrm{CD})$ where the computer randomly decided whether to publicize each subject's guess to the other players. If the missing information, rather than the selective disclosure, has a major impact on observational learning, we should expect a similar treatment effect in the CD treatment. We next report the findings from this new treatment.

\section{Computer Disclosure treatment}

In the computer disclosure treatment $(\mathrm{CD})$, participants were told that the computer would randomly decide whether to disclose their guess to other players. Moreover, as subjects in the OD treatment made the guess and the disclosure decision at the same time, in the $\mathrm{CD}$ treatment, subjects were also informed whether the computer was going to reveal their guess before they made the guess.

To ensure comparability between the OD and CD treatments, for each group in the $\mathrm{CD}$ treatment, the computer implemented the disclosure based on the disclosure pattern obtained from one different group in the OD treatment. That is, the disclosure pattern in the OD and CD treatments was kept identical. For example, suppose for one group in the OD treatment, the first and the second guesses in the first round and the first, the fifth and the ninth guesses in the second round were hidden and all the other guesses were revealed. For the group in the $\mathrm{CD}$ treatment that was randomly assigned to match the disclosure pattern of this group, the guesses would be revealed in exactly the same manner. ${ }^{11}$ Thus, the only difference between the OD and the CD treatment was that in

\footnotetext{
${ }^{11}$ As we try to match the order of the disclosure sequence, strictly speaking, the disclosure is not completely "random". However, since the position of each subject was randomly reassigned in each round, in this sense, whether one's guess is revealed or not is random. The key here is that the disclosure is independent of the nature of the guesses.
} 
the former, the hidden guess was determined by the player and possibly correlated with the player's guess, while in the latter, the hidden guess was determined by the computer independent of the player's guess. As a result of this design, the average disclosure rate was almost identical for the two types: about $72 \%$ of signal consistent guesses and $71 \%$ of signal inconsistent guesses were disclosed. The experiment was otherwise conducted using exactly the same the procedure as the previous two treatments.

Recall that in the OD treatment we find players are less likely to follow the unfavorable information than those in the baseline, which seems to indicate some effect of selective disclosure. However, we observe a similar difference between the CD treatment and the baseline, which implies that this reduced rate of following may largely be due to the missing information effect. Figure 3 reports the information following behavior in the $\mathrm{CD}$ treatment. We find that even in the $\mathrm{CD}$ treatment, the following rate of unfavorable information is reduced to $22.7 \%$. Moreover, about $88.2 \%$ of favorable public information was followed in the $\mathrm{CD}$ treatment, which is lower than the baseline treatment but almost identical to the OD treatment ( $88.2 \%$ vs. 95\%, Mann-Whitney test, $p=0.077 ; 88.2 \%$ vs. $88 \%$, Mann-Whitney test, $p=0.983)$. The data from the CD treatment implies that the missing information effect is dominant in the OD treatment despite of the evidence of selective disclosure.

[Insert Figure 3]

Combined with the theoretical findings, one explanation for the dominant missing information effect is that the values of $k$ and $\delta$ are not high enough for the selectiveness effect to play more than a minor role. There are two possible reasons for the relative low values of $k$ and $\delta$. One is that the role of other-regarding preferences on disclosure choice is limited and many agents are not motivated to help improve others' payoffs. Another possibility is that many agents are motivated to help others, as we hypothesized, but they are unsure as to the most socially efficient way to share the information. While the first explanation suggests that additional incentives should be provided to align the interests of the agents, the second explanation implies that simply providing public information on the most socially efficient information sharing behavior can enhance observational learning. To shed light on these two possible reasons, we conducted a second study to examine the outcome with informed optional disclosure where we promote a shared belief that one can help others by disclosing selectively. 


\section{Informed optional disclosure}

To promote a shared belief of the benefit of selective disclosure, we conducted an informed optional disclosure treatment (IOD). The only difference between the IOD and OD treatments is that we added the following information in the instructions:

"In a previous survey, when people were asked what strategy of guess revealing could help subsequent players make correct guess, the most popular answer was to disclose the guess if and only if it was the same as the color of the private draw."

As in the first study, to shed light on the effect of selectiveness, we also include another computer disclosure treatment (CD_IOD) where the disclosure is implemented by the computer, but the disclosure pattern is matched with the IOD treatment.

If the information manipulation in the IOD treatment leads to more informative and selective disclosure decisions (i.e., a higher $k$ and $\delta$ ), this would suggest that the lack of knowledge of what one should do to help others contributes to the insufficient selectiveness in the OD treatment. In this case, by comparing the IOD and CD_IOD treatments, we can also learn whether the improved selectiveness indeed leads to a higher accuracy rate. On the other hand, if the information manipulation does not make any difference, this will suggest that participants were not motivated to use the disclosure option to help others. Note that, instead of telling one what he/she should do to help others, we try our best to keep the information message neutral by simply providing some factual information about what strategy others think could help the subsequent players. If even such a weak information manipulation leads to a big difference, it provides strong evidence that the lack of knowledge is likely to be the main reason for the insufficient selectiveness.

The experiment procedure was exactly the same as the first study. We ran four sessions of each treatment. Each session involved 20 participants. We first report the disclosure decisions in the IOD treatment. Then we compare the observational learning outcomes among treatments. 


\subsection{Disclosure decisions in the IOD treatment}

In the IOD treatment, subjects were significantly more likely to disclose the signal consistent guesses than the signal inconsistent ones (82.9\% vs. 32.1\%, Mann-Whitney test, $p<0.01$ ). Compared with the OD treatment, in the IOD treatment, the disclosure rate for the signal inconsistent guesses is significantly lower $(32.1 \%$ vs. $48.6 \%$, MannWhitney test, $p=0.05$ ) while the rate for the signal consistent guesses is higher although the difference is not significant probably due to the fact that the rate is already high in the OD treatment ( $82.9 \%$ vs. $76.8 \%$, Mann-Whitney test, $p=0.11)$. These results suggest that the information indeed improves the selectiveness of the disclosure decisions.

To test the treatment difference in the selectiveness between the OD and the IOD treatments, we report in Table 3 the results of a random effect probit regression of the disclosure decisions in the two treatments. The dependent variable is whether the player disclosed his/her guess. The independent variables include: Round $(=1, \ldots, 10)$; $\operatorname{Position}(=1, \ldots, 10)$ of the player; IOD treatment dummy; Signal_inconsistent $(=1$ if the guess is inconsistent with the private signal; $=0$, otherwise); and the interaction between IOD and Signal_inconsistent. As shown in Table 3, the coefficient of the interaction term $\left(\beta_{4}\right)$ is significantly negative, which means the IOD treatment significantly promotes the selectiveness of the disclosure decisions.

\section{[Insert Table 3]}

To provide further information on the differences of disclosure information between the IOD and OD treatments, we report individual types of disclosure behavior in Table 4. Comparing the data reported in Table 2 and Table 4, we find that IOD treatment increases the proportion of selective disclosing subjects dramatically $(66.25 \%$ vs. $38.75 \%$, Z-test, $p<0.01$ ). Interestingly, the increased number of these subjects seems to mostly come from the decrease in the number of random disclosing subjects. This suggests that information manipulation can influence the disclosure patterns of those who are willing to help others, but don't know the correct strategy. Meanwhile, the number of subjects who disclose guesses selectively in an opposite direction remains almost unchanged. This is consistent with our speculation that the disclosure decisions of $\delta<0$ type is probably motivated by self-image. The information manipulation is 
thus not effective if one's disclosure decision is mainly driven by the image concern, rather than the lack of knowledge how to help others.

\section{[Insert Table 4]}

\subsection{Observational learning}

Similar to Figure 2, we report in Figure 4 the frequency of following each type of the information in the IOD treatment and compare it with the baseline, the OD and the $C_{-}$IOD treatments. Compared with the OD treatment where the frequency of following both types of information is lower than the baseline, the IOD treatment lowers the frequency of following the unfavorable information (26.3\% vs. 39.3\%, MannWhitney test, $p<0.01$ ) without the cost of lower following rate of the favorable information ( $96.6 \%$ vs. $95 \%$, Mann-Whitney test, $p=0.22$ ). Data from the CD_IOD treatment demonstrates further that such an improved learning outcome in the IOD treatment is mainly due to the higher selectiveness in the disclosure decision rather than pure change of information amount. To see this, note that Figure 4 shows that the pattern of information following behavior in the CD_IOD treatment is very similar to the baseline treatment $(95.8 \%$ vs. $95 \%$, Mann-Whitney test, $\mathrm{p}=0.62 ; 34.1 \%$ vs. $39.3 \%$, Mann-Whitney test, $\mathrm{p}=0.23$ ).

It is interesting that, compared with the OD treatment, the willingness to follow the public information (both favorable and unfavorable) seems to increase in general in the IOD treatment (96.6\% vs. $88 \%$, Mann-Whitney test, $p<0.01 ; 26.3 \%$ vs. $15.4 \%$, MannWhitney test, $p<0.01)$. An interpretation of this result is that participants become more willing to "trust" the public information in the IOD treatment. The increased probability of following unfavorable public information can diminish the accuracy. However, as we explained above, the overall quality of the guesses relies more on following favorable public information than abandoning unfavorable information. Indeed, using the same accuracy rate measurement as defined in section 5.2 we find IOD treatment achieves a higher accuracy rate than the OD treatment $(86.1 \%$ vs. $76.5 \% t$-test, $p=0.04)$. The accuracy rate in the IOD is also higher than the baseline and the CD_IOD treatment although the difference is not significant or marginal significant (IOD vs. baseline: $86.1 \%$ vs. $78.2 \%, t$-test, $p=0.13$; IOD vs. CD_IOD: $86.1 \%$ vs. $76.7 \%, t$-test, $p=$ 0.07). 


\section{[Insert Figure 4]}

Taking all the above results together, the additional information provided in the IOD treatment helps other-regarding subjects to identify the socially beneficial disclosure strategy and consequently increases the efficiency of utilizing the public information in decision making.

\section{Conclusion}

Previous research on observational learning has assumed that individuals do not have discretion regarding the visibility of their decisions. We investigate how optional disclosure may affect the outcome of observational learning. We identify theoretically the conditions under which optional disclosure can improve or degrade observational learning. Our experiments provide empirical evidence regarding individual information sharing decisions.

In the traditional observational learning setting, helping subsequent players is costly, as one needs to always make the guess based on her own private signal even though she is better off by herding. In contrast, optional disclosure removes the cost of helping the subsequent decision-makers. The reason is that a person can use the disclosure decisions to signal the private information and make the guess to the best of his/her interest (e.g., by herding). It is thus more likely for other-regarding preference to play a role in the outcome of observational learning. Our experimental data show that a significant number of individuals exhibit disclosure behavior consistent with the other-regarding preference hypothesis, in that they reveal the guesses selectively. Nevertheless, the consequent benefit of selective disclosure is not sufficient to overcome the negative impact of missing information on observational learning. As a result, optional disclosure does not improve and may even lower the overall decision quality.

Our findings suggest that in the context of observational learning, it is important to consider the factors underlying decisions to reveal one's own behavior. When agents have discretion regarding whether to disclose, additional institutions may be necessary to achieve efficient outcomes. We have shown in this paper that it can be beneficial to simply provide additional, albeit suggestive, information regarding how one's disclosure behavior can help others. In future research it would be beneficial to 
test and compare alternative mechanisms to achieve this goal. 


\section{References}

Acemoglu, D., Dahleh, M. A., Lobel, I., \& Ozdaglar, A. (2011). Bayesian learning in social networks. The Review of Economic Studies, 78(4), 1201.

Alevy, J. E., Haigh, M. S., \& List, J. A. (2007). Information cascades: Evidence from a field experiment with financial market professionals. The Journal of Finance, 62(1), $151-180$.

Ali, S. N. \& Kartik, N. (2012). Herding with collective preferences. Economic Theory, 51(3), 601-626.

Anderson, L. R. \& Holt, C. A. (1997). Information cascades in the laboratory. The American Economic Review, 87(5), 847-862.

Andreoni, J. \& Miller, J. (2002). Giving according to garp: An experimental test of the consistency of preferences for altruism. Econometrica, 70(2), 737-753.

Ash, E. \& Van Parys, J. (2015). Group identity bias and information cascades. Available at http://elliottash.com/wp-content/uploads/2013/08/Ash-VanParys-2015.pdf.

Banerjee, A. V. (1992). A simple model of herd behavior. The Quarterly Journal of Economics, 107(3), 797.

Bikhchandani, S., Hirshleifer, D., \& Welch, I. (1992). A theory of fads, fashion, custom, and cultural change as informational cascades. Journal of Political Economy, 100(5), 992-1026.

Bikhchandani, S., Hirshleifer, D., \& Welch, I. (1998). Learning from the behavior of others: Conformity, fads, and informational cascades. The Journal of Economic Perspectives, 12(3), 151-170.

Çelen, B. \& Hyndman, K. (2012). Social learning through endogenous information acquisition: An experiment. Management Science, 58(8), 1525-1548.

Celen, B., Kariv, S., \& Schotter, A. (2010). An experimental test of advice and social learning. Management Science, 56(10), 1687-1701. 
Charness, G. \& Rabin, M. (2002). Understanding social preferences with simple tests. The Quarterly Journal of Economics, 117(3), 817-869.

Cipriani, M. \& Guarino, A. (2005). Herd behavior in a laboratory financial market. The American Economic Review, 95(5), 1427-1443.

Cooper, D. \& Kagel, J. H. (2015). Other-regarding preferences: A selective survey of experimental results. In The Handbook of Expeirmental Economics, volume 2 (pp. 217-289). Princeton University Press.

Davis, B. J. (2017). An experiment on behavior in social learning games with collective preferences. Economics Letters, 152, 93-95.

Diamond, D. W. \& Dybvig, P. H. (1983). Bank runs, deposit insurance, and liquidity. Journal of Political Economy, 91(3), 401-419.

Drehmann, M., Oechssler, J., \& Roider, A. (2005). Herding and contrarian behavior in financial markets: An internet experiment. The American Economic Review, 95(5), 1403-1426.

Fahr, R. \& Irlenbusch, B. (2011). Who follows the crowd - groups or individuals? Journal of Economic Behavior \& Organization, 80(1), 200.

Fehr, E. \& Fischbacher, U. (2003). The nature of human altruism. Nature, 425(6960), $785-791$.

Fehr, E. \& Schmidt, K. M. (1999). A theory of fairness, competition, and cooperation. The Quarterly Journal of Economics, 114(3), 817-868.

Fischbacher, U. (2007). Z-tree: Zurich toolbox for ready-made economic experiments. Experimental Economics, 10(2), 171-178.

Goeree, J. K., Palfrey, T. R., Rogers, B. W., \& McKelvey, R. D. (2007). Self-correcting information cascades. The Review of Economic Studies, 74(3), 733-762.

Goeree, J. K. \& Yariv, L. (2015). Conformity in the lab. Journal of the Economic Science Association, 1(1), 15-28. 
Hung, A. A. \& Plott, C. R. (2001). Information cascades: Replication and an extension to majority rule and conformity-rewarding institutions. The American Economic Review, 91(5), 1508-1520.

Kübler, D. \& Weizsäcker, G. (2004). Limited depth of reasoning and failure of cascade formation in the laboratory. The Review of Economic Studies, 71(2), 425-441.

Kultti, K. \& Miettinen, P. (2006). Herding with costly information. International Game Theory Review, 08(01), 21-31.

Kultti, K. \& Miettinen, P. (2007). Herding with costly observation. The BE Journal of Theoretical Economics, 7(1), 1-16.

Lee, Y. J., Hosanagar, K., \& Tan, Y. (2015). Do I follow my friends or the crowd? Information cascades in online movie ratings. Management Science, 61(9), 22412258.

Lobel, I. \& Sadler, E. (2015). Information diffusion in networks through social learning. Theoretical Economics, 10(3), 807-851.

March, C. \& Ziegelmeyer, A. (2016). Altruistic observational learning. CESifo Working Paper Series No. 5792. Available at SSRN: https://ssrn.com/abstract=2760259.

Nöth, M. \& Weber, M. (2003). Information aggregation with random ordering: Cascades and overconfidence. The Economic Journal, 113(484), 166-189.

Smith, L. \& Sorensen, P. N. (2008). Rational social learning by random sampling. Available at SSRN: https://ssrn.com/abstract $=1138095$.

Song, Y. (2016). Social learning with endogenous observation. Journal of Economic Theory, 166, 324-333.

Weizsäcker, G. (2010). Do we follow others when we should? A simple test of rational expectations. The American Economic Review, 100(5), 2340-2360.

Zhang, M. (2015). Essays in Social Learning. PhD thesis, The London School of Economics and Political Science. 
Ziegelmeyer, A., Koessler, F., Bracht, J., \& Winter, E. (2010). Fragility of information cascades: an experimental study using elicited beliefs. Experimental Economics, 13(2), 121-145. 

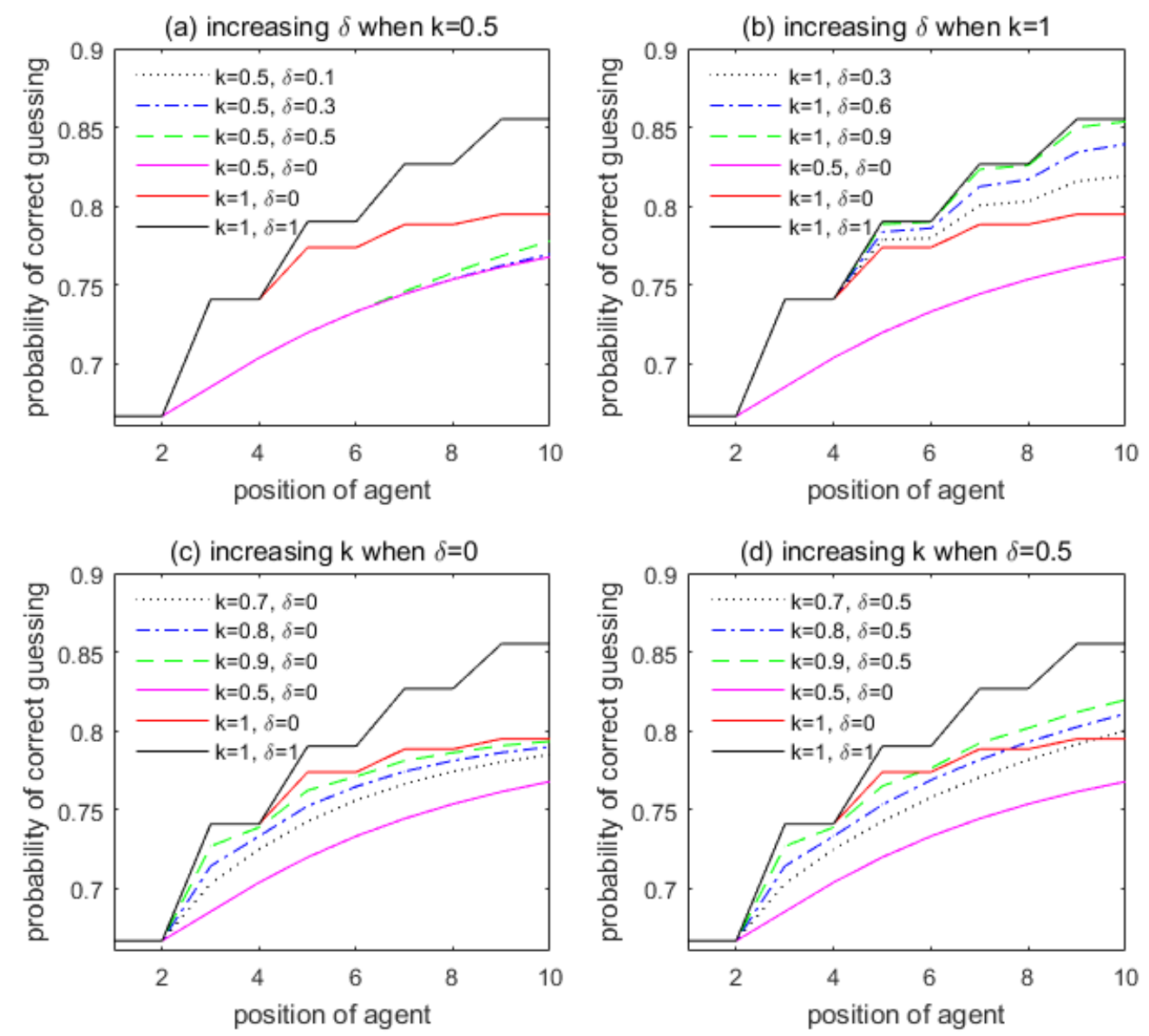

Figure 1: Numeric simulation for the probability of correct guessing under various parametric settings 


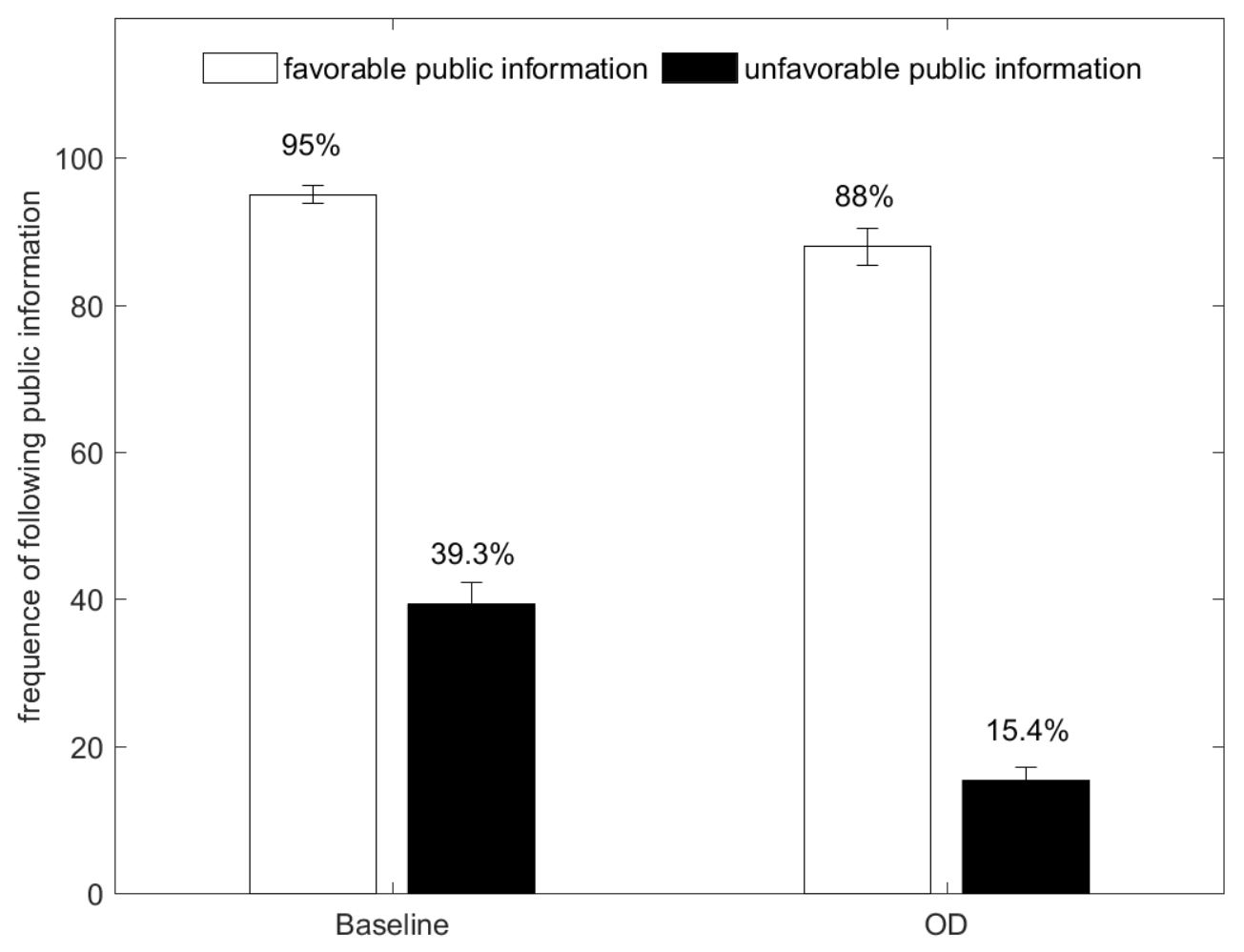

Figure 2: Frequency of following public information by treatments 


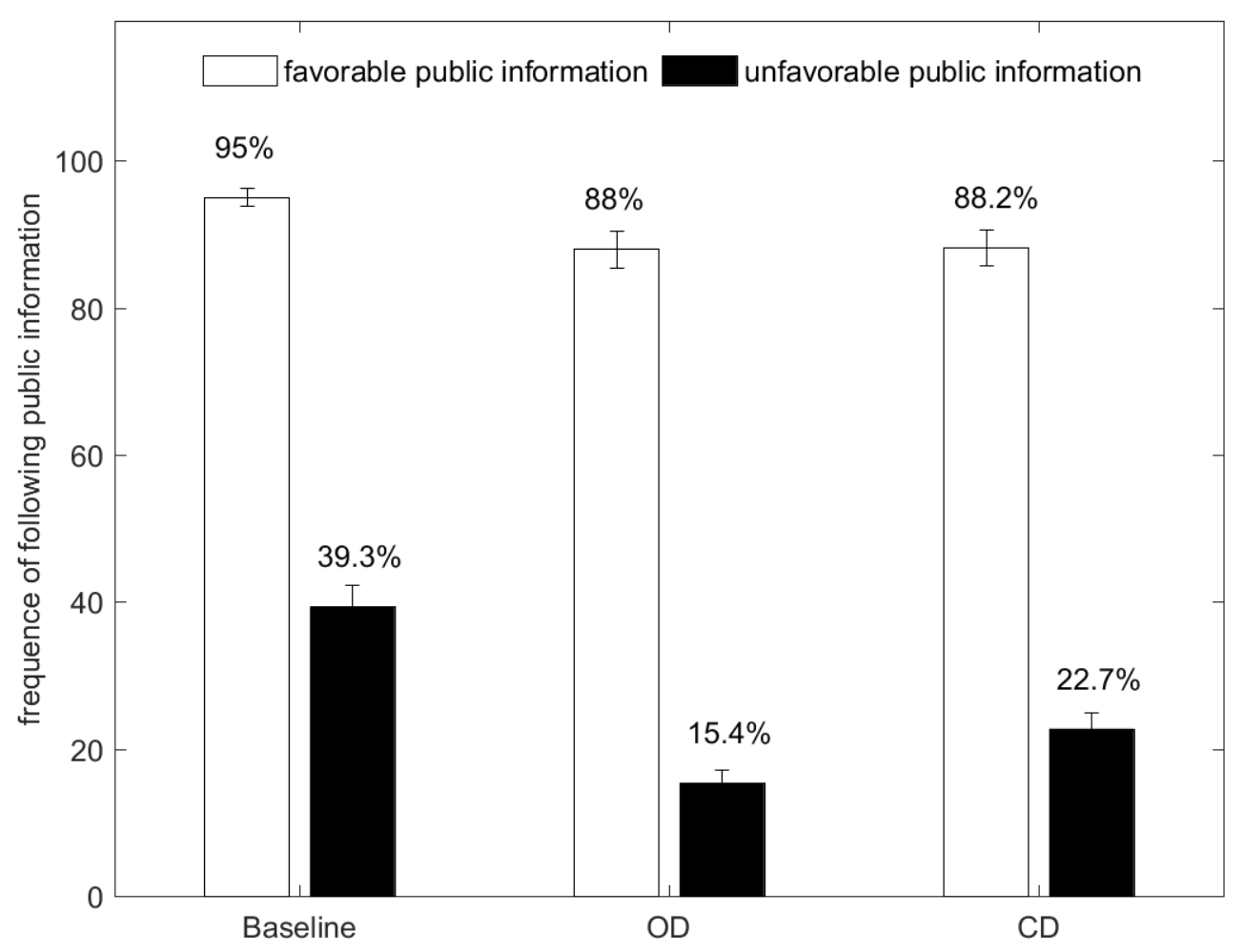

Figure 3: Frequency of following public information by treatments 


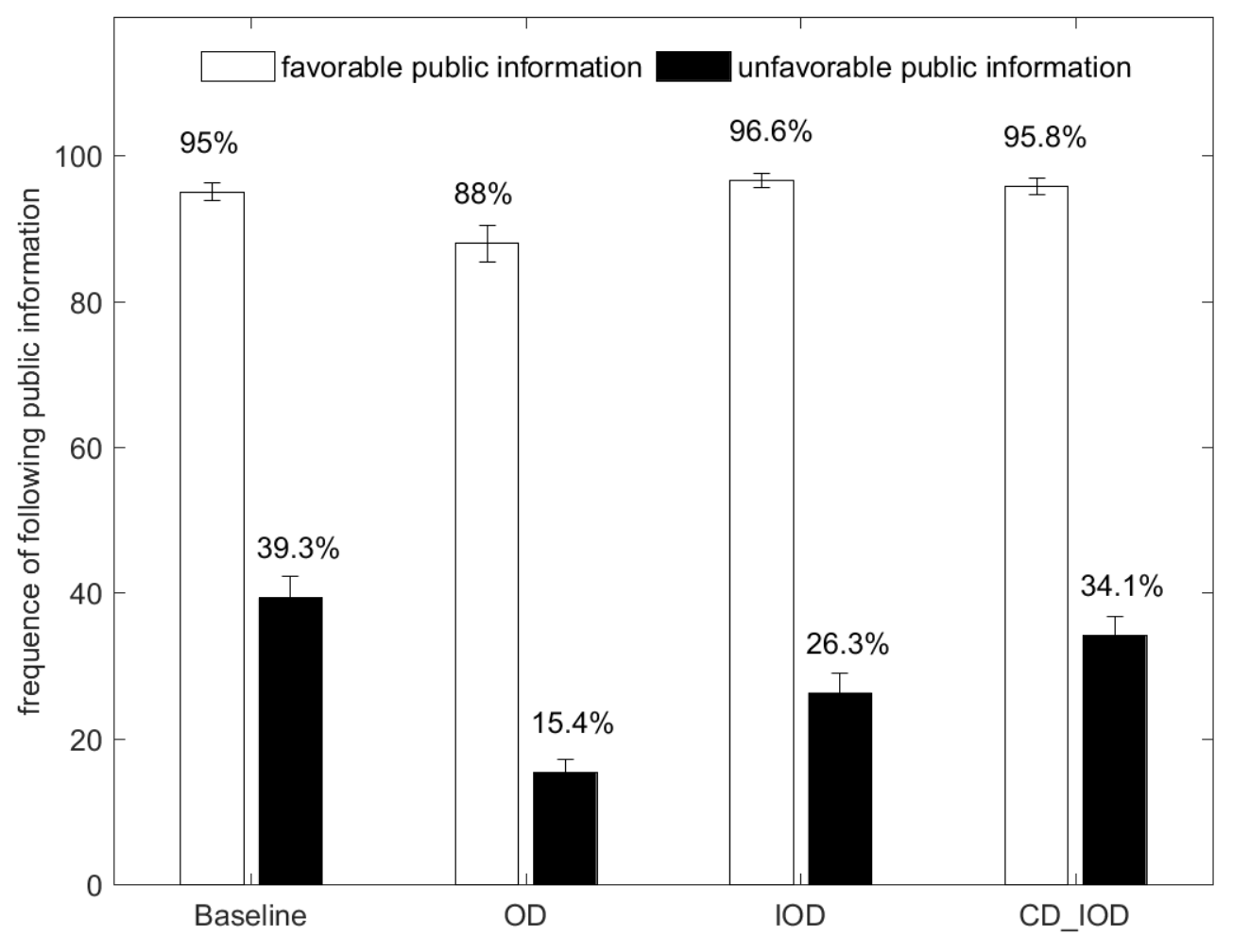

Figure 4: Frequency of following the public information by treatments 
Table 1: Random effect probit regression analysis of disclosure decisions in the OD treatment

\begin{tabular}{lc}
\hline & \multicolumn{2}{c}{ Independent variable: } \\
& $d_{r, t}=1$ if in round $r$ the player $t$ disclose his/her guess; \\
& $=0$ o.w. \\
\hline$\beta_{1}:$ Round $(r)$ & $-0.029(0.025)$ \\
$\beta_{2}:$ Position $(t)$ & $0.064^{* * *}(0.022)$ \\
$\beta_{3}:$ Signal inconsistent & $-1.2^{* *}(0.215)$ \\
Constant & $0.759^{*}(0.210)$ \\
Log likelihood & -362.3 \\
\# of Obs. & 716 \\
\hline
\end{tabular}

Note: ${ }^{* *}$ : significant at the $1 \%$ level; ${ }^{* *}$ : significant at the $5 \%$ level. The numbers in the parenthesis are the robust standard errors. 
Table 2: Individual disclosure patterns

\begin{tabular}{lrr}
\hline Individual disclosure pattern & $\begin{array}{c}\text { \# of } \\
\text { subjects }\end{array}$ & $\begin{array}{c}\% \text { of } \\
\text { subjects }\end{array}$ \\
\hline A. Make both types of guesses & $\mathbf{6 0}$ & $\mathbf{7 5 . 0 0 \%}$ \\
A1. Disclose consistent guesses more frequently $(\delta>0)$ & 31 & $38.75 \%$ \\
: Disclose more than half of the consistent guesses $(\delta>0 \& k>0.5)$ & 27 & $33.75 \%$ \\
: Disclose no more than half the consistent guesses $(\delta>0 \& k \leq 0.5)$ & 4 & $5.00 \%$ \\
A2. Disclose inconsistent guesses more frequently $(\delta<0)$ & 15 & $18.75 \%$ \\
: Disclose more than half of the consistent guesses $(\delta<0 \& k>0.5)$ & 11 & $13.75 \%$ \\
: Disclose no more than half the consistent guesses $(\delta<0 \& k \leq 0.5)$ & 4 & $5 \%$ \\
A3. Disclose both types equal frequently $(\delta=0)$ & 14 & $17.50 \%$ \\
: Disclose all of them $(\delta=0 \& k=1)$ & 11 & $13.75 \%$ \\
: Disclose half of them $(\delta=0 \& k=0.5)$ & 2 & $2.50 \%$ \\
: Disclose less half of them $(\delta=0 \& 0<k<0.5)$ & 1 & $1.25 \%$ \\
& & \\
B. Only make consistent guesses & $\mathbf{2 0}$ & $\mathbf{2 5 . 0 0 \%}$ \\
B1. Disclose more than half of them $(k>0.5)$ & 12 & $15.00 \%$ \\
: Disclose all the consistent guesses $(k=1)$ & 6 & $7.50 \%$ \\
: Disclose more than half but not all $(0.5<k<1)$ & 6 & $7.50 \%$ \\
B2. Disclose half of them $(k=0.5)$ & 5 & $6.25 \%$ \\
B3. Disclose less than half $(k<0.5)$ & 3 & $3.75 \%$ \\
\hline
\end{tabular}


Table 3: Random effect probit regression analysis of disclosure decisions in the OD and IOD treatments

\begin{tabular}{lc}
\hline & $\begin{array}{c}\text { Independent variable: } \\
d_{r, t}=1 \text { if in round } r \text { the player } t \text { disclose his/her guess; } \\
=0 \text { o.w. }\end{array}$ \\
\hline$\beta_{1}$ : IOD & $0.217^{*}$ \\
$\beta_{2}$ : Round $(r)$ & $(0.152)$ \\
$\beta_{3}$ : Position $(t)$ & -0.008 \\
& $(-0.018)$ \\
$\beta_{4}$ : Signal inconsistent & $0.0263^{*}$ \\
$\beta_{5}$ : Signal inconsistent $*$ IOD & $(0.016)$ \\
& $-0.994^{* *}$ \\
Constant & $(0.182)$ \\
& $-0.698^{* * *}$ \\
Log likelihood & $(0.261)$ \\
\# of Obs. & $-0.716^{* * *}$ \\
\end{tabular}

Note: ${ }^{* *}$ : significant at the $1 \%$ level; ${ }^{* *}$ : significant at the $5 \%$ level. The numbers in the parenthesis are the robust standard errors. 
Table 4: Individual disclosure patterns

\begin{tabular}{lrr}
\hline Individual disclosure pattern & $\begin{array}{c}\text { \# of } \\
\text { subjects }\end{array}$ & $\begin{array}{c}\text { \% of } \\
\text { subjects }\end{array}$ \\
\hline A. Make both types of guesses & $\mathbf{7 0}$ & $\mathbf{8 7 . 5 0} \%$ \\
A1. Disclose consistent guesses more frequently $(\delta>0)$ & 53 & $66.25 \%$ \\
: Disclose more than half of the consistent guesses $(\delta>0 \& k>0.5)$ & 50 & $62.50 \%$ \\
: Disclose no more than half of consistent guesses $(\delta>0 \& k \leq 0.5)$ & 3 & $3.75 \%$ \\
A2. Disclose inconsistent guesses more frequently $(\delta<0)$ & 13 & $16.25 \%$ \\
: Disclose more than half of the consistent guesses $(\delta<0 \& k>0.5)$ & 11 & $13.75 \%$ \\
: Disclose no more than half the consistent guesses $(\delta<0 \& k \leq 0.5)$ & 2 & $2.5 \%$ \\
A3. Disclose both types equal frequently $(\delta=0)$ & 4 & $5.00 \%$ \\
: Disclose all of them $(\delta=0 \& k=1)$ & 4 & $5.00 \%$ \\
& & \\
B. Only make consistent guesses & $\mathbf{1 0}$ & $\mathbf{1 2 . 5 0 \%}$ \\
B1. Disclose more than half of them $(k>0.5)$ & 6 & $7.50 \%$ \\
: Disclose all the consistent guesses $(k=1)$ & 1 & $1.25 \%$ \\
: Disclose more than half but not all $(0.5<k<1)$ & 5 & $6.25 \%$ \\
B2. Disclose half of them $(k=0.5)$ & 2 & $2.50 \%$ \\
B3. Disclose less than half $(k<0.5)$ & 2 & $2.50 \%$ \\
\hline
\end{tabular}




\section{Appendix A Instructions (Translated from Chinese)}

Below are the instructions of OD, CD , IOD and CI_IOD treatments. The instructions for the Baseline treatment differs from the OD treatment only in that participants are not asked to decide whether to reveal the guess and other explanations related to this difference.

\section{All four treatments:}

Welcome to the experiment. This research is supported by National Natural Science Foundation of China. You have earned 15 RMB for showing up on time. In addition, you may earn more money depending on your decisions during the experiment. Please read the following instructions carefully.

This experiment consists of one practice round, which will not affect your final earnings, followed by 10 paying rounds, which will determine your final earnings. You should finish the decision task in each round.

At the beginning of each round, the computer will randomly form two groups of ten participants. In each group, the ten participants will do the task sequentially and the order is randomly determined by the computer. All decisions are anonymous. You will not be informed of the identities of any other participants in your group throughout the experiment.

Below we describe the decision task:

1. Imagine two urns with otherwise identical appearance. The "RED" urn contains two RED balls and one WHITE ball; and the "WHITE" urn contains two WHITE balls and one RED ball.

2. At the beginning of each round, the computer randomly selects one urn with an equal probability. Participants do not know which urn is chosen until the end of the experiment. The chosen urn will be used throughout this round.

3. A participant's decision task in a round is to guess the color of the urn (White or Red). The procedure is as follows:

\section{$O D$ and IOD treatments:}

First, the computer randomly draws a ball from the urn, shows its color on the first player's screen and then returns it to the urn. For example, if a white ball is drawn, 
the first player's screen displays: "the randomly drawn ball: white ball." This is private information for the first player; any other participants in the group will not know the color of the drawn ball. The first player will then 1) guess the color of the urn; 2) decide whether to reveal his/her guess. After he/she has submitted the two decisions and if he/she has decided to reveal, his/her guess will be displayed on the screens of all other nine participants in the group. For example, if the first player's guess is WHITE urn and if he/she has decided to reveal the guess, the screens of other participants show: "the first player's guess: WHITE urn." If the first player decides not to reveal, the screens of other participants show: "the first player's guess: not revealed."

Next, the computer makes another draw from the same urn, shows the color of the drawn ball on the second player's screen and then returns it to the urn. Again, this is private information for the second player. In addition, as described above, if the first player has decided to reveal the guess, the second player also observes the first player's guess. The second player will then 1) guess the color of the urn; 2) decide whether to reveal his/her guess. After he/she has submitted the two decisions and if he/she has decided to reveal, his/her guess will be displayed on the screens of all other nine participants in the group.

The same procedure applies to all other players. Each player receives the following information before making the decisions: the urn guesses of all the preceding players who have decided to reveal their guesses; and the color of the ball privately drawn by the computer. Note that, every ball is drawn from the same urn and every ball is returned to the urn before the next draw. A participant will not be able to change his/her decisions once submitted. At the end of the experiment, participants will be informed of the true color of the urn in each round.

4. A round ends when the last (tenth) player has submitted the decisions. The compute will randomly re-group participants and determine the order of decisionmaking in each group in a new round. A new round then begins and follows the same procedure.

5. After each round, please record on the "Experiment Record Sheet":

1) the revealed urn guesses of all other participants in your group;

2) your order of decision-making; 
3) the color of the ball privately drawn for you;

4) your own guess;

5) your decision whether to reveal your guess. At the end of the experiment, please record the true color of the urn in each round and whether your guess in this round is correct or not in the last two rows of the "Experiment Record Sheet."

\section{$C D$ and $C D_{-} I O D$ treatments:}

First, the first player is informed whether his/her guess will be revealed to all other players in the group. This revealing decision is randomly determined by the computer. Next, the computer randomly draws a ball from the urn, shows its color on the first player's screen and then returns it to the urn. For example, if a white ball is drawn, the first player's screen displays: "the randomly drawn ball: white ball." This is private information for the first player; any other participants in the group will not know the color of the drawn ball. The first player will then guess the color of the urn. After he/she submits the guess and if his/her guess is to be revealed, his/her guess will be displayed on the screens of all other nine participants in the group. For example, if the first player's guess is WHITE urn and if his/her guess is to be revealed, the screens of other participants show: "the first player's guess: WHITE urn.” If his/her guess is not to be revealed, the screens of other participants show: "not revealed."

Next, the computer makes another draw from the same urn, shows the color of the drawn ball on the second player's screen and then returns it to the urn. Again, this is private information for the second player. In addition, as described above, if the computer has randomly determined to reveal the first player's guess, the second player also observes the first player's guess. The second player will then guess the color of the urn. After he/she has submitted the urn guess and if his/her guess is to be revealed, his/her guess will be displayed on the screens of all other nine participants in the group.

The same procedure applies to all other players. Each player receives the following information before making the guess: the urn guesses of all the preceding players revealed by the computer; the color of the ball privately drawn by the computer; and whether the computer will reveal his/her guess to other participants in the group. Note that, every ball is drawn from the same urn and every ball is returned to the urn before the next draw. A participant will not be able to change his/her guess once submitted. 
At the end of the experiment, participants will be informed of the true color of the urn in each round.

4. A round ends when the last (tenth) player submits the guess. The compute will randomly re-group participants and determine the order of decision-making in each group in a new round. A new round then begins and follows the same procedure.

5. After each round, please record on the "Experiment Record Sheet":

1) the revealed urn guesses of all other participants in your group;

2) your order of decision-making;

3) the color of the ball privately drawn for you;

4) your own guess;

5) whether your guess was revealed by the computer. At the end of the experiment, please record the true color of the urn in each round and whether your guess in this round is correct or not in the last two rows of the "Experiment Record Sheet."

\section{$I O D$ and $C D_{-} I O D$ treatments:}

NOTE: In a previous survey, when people were asked what strategy of guess revealing could help subsequent players make correct guesses, the most popular answer was to reveal the guess if and only if it was the same as the color of the private draw.

\section{All four treatments:}

\section{Earnings}

Your earnings from the experiment have two components:

1. Show-up bonus: $15 \mathrm{RMB}$

2. Additional earnings: at the end of the experiment, the experimenter will randomly select one out of ten paying rounds. If you have made a correct urn guess in the selected round, you will earn an additional 40 RMB. If you have made an incorrect guess, you will receive no additional earnings. 


\section{Appendix B Simulation Results}
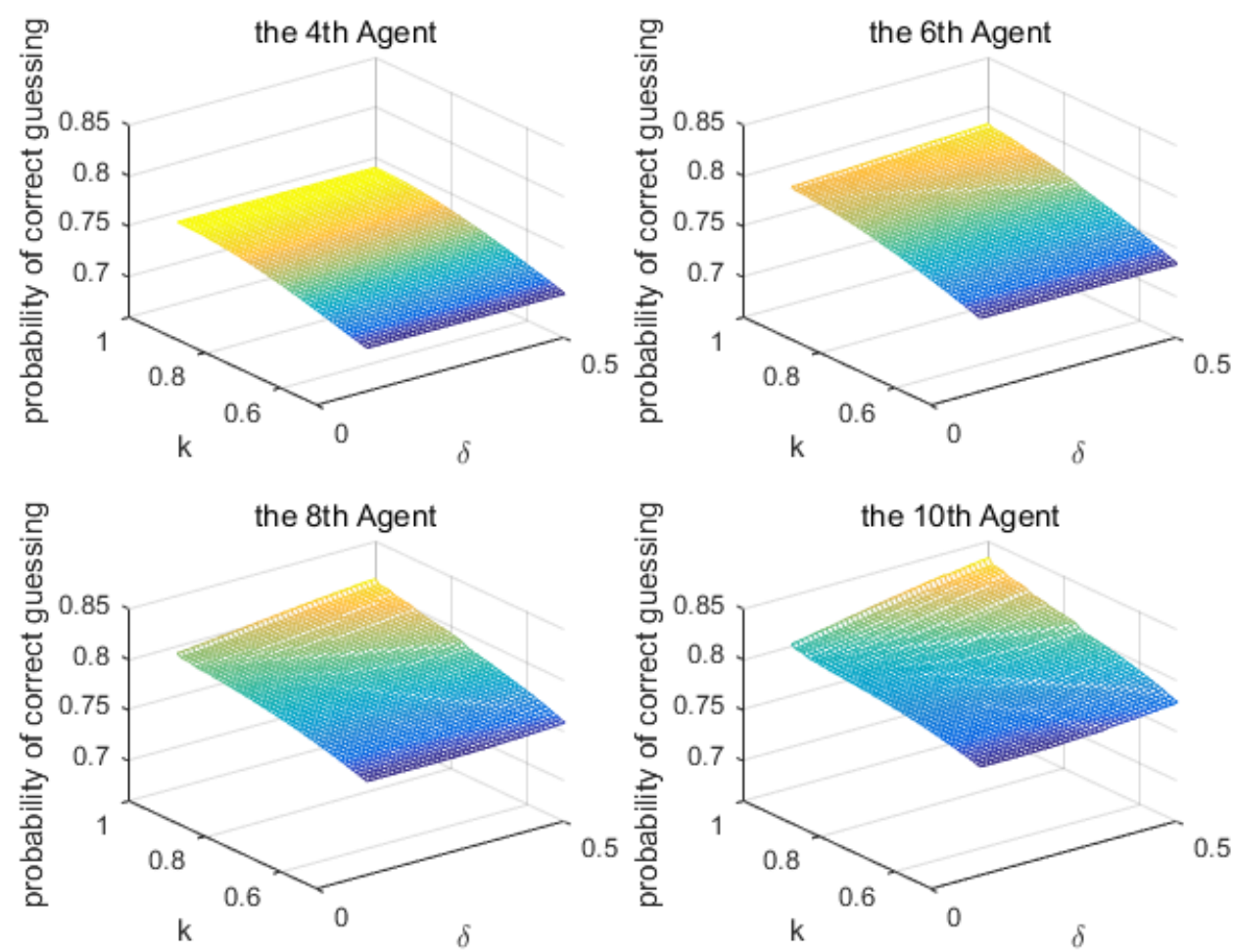


\section{Appendix C Mathematical Proofs}

Lemma C.1. Conditional on belief $h_{t}$, the distribution of $h_{t+1}$ is of the following form

(i)

$$
\left\{\begin{array}{l}
\mathbb{P}\left(h_{t+1}=h_{t}+1|| h_{t} \mid \leq 1\right)=q k \\
\mathbb{P}\left(h_{t+1}=h_{t}-1|| h_{t} \mid \leq 1\right)=(1-q) k \\
\mathbb{P}\left(h_{t+1}=h_{t}|| h_{t} \mid \leq 1\right)=1-k
\end{array}\right.
$$

(ii)

$$
\left\{\begin{array}{l}
\mathbb{P}\left(h_{t+1}=h_{t}+\theta \mid h_{t}>1, d_{t}=1\right)=q_{\theta}, \\
\mathbb{P}\left(h_{t+1}=h_{t}-\theta \mid h_{t}<-1, d_{t}=1\right)=\lambda q_{\theta}, \\
\mathbb{P}\left(h_{t+1}=h_{t}-\eta \mid h_{t}>1, d_{t}=0\right)=1-q_{\theta}, \\
\mathbb{P}\left(h_{t+1}=h_{t}+\eta \mid h_{t}<-1, d_{t}=0\right)=1-\lambda q_{\theta},
\end{array}\right.
$$

where

$$
\left\{\begin{array}{lll}
\theta=\log _{\frac{q}{1-q}}\left[\frac{k-\delta+\delta q}{k-\delta q}\right], & & \theta \in(0,1], \\
\eta=\log _{\frac{q}{1-q}}\left[\frac{1-k+\delta q}{1-k+\delta(1-q)}\right], & & \eta \in(\theta, 1], \\
q_{\theta}=k-\delta+\delta q, & \lambda \in(0,1] . \\
\lambda=\left(\frac{q}{1-q}\right)^{-\theta}, & \lambda \in P^{-\theta}
\end{array}\right.
$$

The characteristics of belief path will be used to prove Proposition 1 and Proposition 2.

Proof. (i) Before information cascade $\left(\left|h_{t}\right| \leq 1\right)$, an agent will follow the private signal that she received, that is, $a_{t}=s_{t}$. If the agent $t$ chooses to disclose her decision $\left(d_{t}=1\right)$, the public belief $h_{t+1}$ at time $t+1$ is

$$
\begin{aligned}
h_{t+1} & =\log _{\frac{q}{1-q}}\left[\frac{\mathbb{P}\left(H_{t+1} \mid \omega=1\right)}{\mathbb{P}\left(H_{t+1} \mid \omega=-1\right)}\right] \\
& =\log _{\frac{q}{1-q}}\left[\frac{\mathbb{P}\left(H_{t}, d_{t} a_{t} \mid \omega=1\right)}{\mathbb{P}\left(H_{t} d_{t} a_{t} \mid \omega=-1\right)}\right] \\
& =\log _{\frac{q}{1-q}}\left[\frac{\mathbb{P}\left(H_{t}, s_{t} \mid \omega=1\right)}{\mathbb{P}\left(H_{t}, s_{t} \mid \omega=-1\right)}\right] \\
& =\log _{\frac{q}{1-q}}\left[\frac{\mathbb{P}\left(H_{t} \mid \omega=1\right)}{\mathbb{P}\left(H_{t} \mid \omega=-1\right)} \cdot \frac{\mathbb{P}\left(s_{t} \mid \omega=1\right)}{\mathbb{P}\left(s_{t} \mid \omega=-1\right)}\right] \\
& =h_{t}+\log _{\frac{q}{1-q}}\left[\frac{\mathbb{P}\left(s_{t} \mid \omega=1\right)}{\mathbb{P}\left(s_{t} \mid \omega=-1\right)}\right] .
\end{aligned}
$$


Recalling $\mathbb{P}\left(s_{t}=1 \mid \omega=1\right)=\mathbb{P}\left(s_{t}=-1 \mid \omega=-1\right)=q$, we have

$$
h_{t+1}= \begin{cases}h_{t}+1, & \text { if } s_{t}=1, \\ h_{t}-1, & \text { if } s_{t}=-1 .\end{cases}
$$

Correspondingly,

$$
\mathbb{P}\left(h_{t+1}=h_{t}+1|| h_{t} \mid \leq 1\right)=\mathbb{P}\left(d_{t}=1\right) * \mathbb{P}\left(s_{t}=1\right)=q k,
$$

and

$$
\mathbb{P}\left(h_{t+1}=h_{t}-1|| h_{t} \mid \leq 1\right)=\mathbb{P}\left(d_{t}=1\right) * \mathbb{P}\left(s_{t}=-1\right)=(1-q) k
$$

Thus, we have $\mathbb{P}\left(h_{t+1}=h_{t}|| h_{t} \mid \leq 1\right)=1-k$.

Note that $\mathbb{P}\left(s_{t}=1\right)$ is short for $\mathbb{P}\left(s_{t}=1 \mid \omega=1\right)$, which means the signal is consistent with the correct answer of the game.

(ii) If $h_{t}>1$, we have $a_{t}=h_{t} /\left|h_{t}\right|=1$. A disclosure of $a_{t}=1$ actually is consistent with two cases: a disclosure of private-signal consistent guess and a disclosure of conformity decision. That is,

$$
\begin{aligned}
\mathbb{P}\left(a_{t}=1 \mid \omega=1\right) & =\mathbb{P}\left(s_{t}=1 \mid \omega=1\right) * k+\mathbb{P}\left(s_{t}=-1 \mid \omega=1\right) *(k-\delta) \\
& =q * k+(1-q) *(k-\delta) \\
& =k-\delta+\delta q .
\end{aligned}
$$

Following the same lines, we have

$$
\mathbb{P}\left(a_{t}=1 \mid \omega=-1\right)=k-\delta q .
$$

Thus, a disclosure of decision leads to an update of public belief $h_{t+1}$ in the 
following way,

$$
\begin{aligned}
h_{t+1} & =\log _{\frac{q}{1-q}}\left[\frac{\mathbb{P}\left(H_{t+1} \mid \omega=1, h_{t}>1\right)}{\mathbb{P}\left(H_{t+1} \mid \omega=-1, h_{t}>1\right)}\right] \\
& =\log _{\frac{q}{1-q}}\left[\frac{\mathbb{P}\left(H_{t}, d_{t} a_{t} \mid \omega=1, h_{t}>1\right)}{\mathbb{P}\left(H_{t}, d_{t} a_{t} \mid \omega=-1, h_{t}>1\right)}\right] \\
& =\log _{\frac{q}{1-q}}\left[\frac{\mathbb{P}\left(H_{t}, a_{t} \mid \omega=1, h_{t}>1\right)}{\mathbb{P}\left(H_{t}, a_{t} \mid \omega=-1, h_{t}>1\right)}\right] \\
& =\log _{\frac{q}{1-q}}\left[\frac{\mathbb{P}\left(H_{t} \mid \omega=1\right)}{\mathbb{P}\left(H_{t} \mid \omega=-1\right)} \frac{\mathbb{P}\left(a_{t} \mid \omega=1, h_{t}>1\right)}{\mathbb{P}\left(a_{t} \mid \omega=-1, h_{t}>1\right)}\right] \\
& =h_{t}+\log _{\frac{q}{1-q}}\left[\frac{\mathbb{P}\left(a_{t} \mid \omega=1, h_{t}>1\right)}{\mathbb{P}\left(a_{t} \mid \omega=-1, h_{t}>1\right)}\right] \\
& =h_{t}+\log _{\frac{q}{1-q}}\left[\frac{\mathbb{P}\left(a_{t}=1 \mid \omega=1\right)}{\mathbb{P}\left(a_{t}=1 \mid \omega=-1\right)}\right] \\
& =h_{t}+\log _{\frac{q}{1-q}}\left[\frac{k-\delta+\delta q}{k-\delta q}\right] \\
& =h_{t}+\theta, \quad
\end{aligned}
$$

where $\theta=\log _{\frac{q}{1-q}}\left[\frac{k-\delta+\delta q}{k-\delta q}\right], \theta \in(0,1)$.

Obviously, we have

$$
\mathbb{P}\left(h_{t+1}=h_{t}+\theta \mid h_{t}>1\right)=\mathbb{P}\left(a_{t}=1 \mid \omega=1\right)=k-\delta+\delta q .
$$

The first equation (C.2) in the distribution is proved. The other equations in (C.2) can be proved along similar lines.

\section{Proof for Proposition 1.}

The statements in Proposition 1 will be proved by the induction method.

Proof. (i) To prove the first statement, we define

$$
f_{t}(k):=\mathbb{P}\left(\left|h_{t}\right|>1 \mid k, \delta=0\right) .
$$

The first statement is equivalent to the case that $f_{t}$ is an increasing function on $[0,1]$ for any $t \in\{2, \ldots, T\}$.

At time $t=2$, we have $f_{2}(k)=k^{2}\left(q^{2}+(1-q)^{2}\right)$. Obviously, $f_{2}$ is an increasing function on $[0,1]$. Assuming $f_{t_{0}}$ is an increasing function, we are going to prove $f_{t_{0}+1}$ is an increasing function as well. Then, by the induction method, the first statement will be proved. 
At time $t_{0},\left(t_{0} \in\{2, \ldots, T\}\right)$, the public belief $h_{t_{0}}$ has five states, i.e., $\{-2,-1,0,1,2\}$. The distributions at time $t_{0}$ and $t_{0}+1$ are given in the following way

\begin{tabular}{llccccc}
\hline & state $i$ & -2 & -1 & 0 & 1 & 2 \\
\hline$t=t_{0}$ & $\mathbb{P}\left(h_{t_{0}}=i \mid 0<k \leq 1, \delta=0\right)$ & $p_{1}$ & $p_{2}$ & $p_{3}$ & $p_{4}$ & $p_{5}$ \\
$t=t_{0}+1$ & $\mathbb{P}\left(h_{t_{0}+1}=i \mid 0<k \leq 1, \delta=0\right)$ & $\tilde{p}_{1}$ & $\tilde{p}_{2}$ & $\tilde{p}_{3}$ & $\tilde{p}_{4}$ & $\tilde{p}_{5}$ \\
\hline
\end{tabular}

Then, we have

$$
f_{t_{0}}(k)=p_{1}+p_{5}=1-\left(p_{2}+p_{3}+p_{4}\right),
$$

and

$$
f_{t_{0}+1}(k)=\tilde{p}_{1}+\tilde{p}_{5}=1-\left(\tilde{p}_{2}+\tilde{p}_{3}+\tilde{p}_{4}\right) .
$$

From the path property of public belief (Lemma C.1), we have

$\tilde{p}_{2}+\tilde{p}_{3}+\tilde{p}_{4}=p_{2}(1-(1-q) k)+p_{3}+p_{4}(1-q k)=\left(p_{2}+p_{3}+p_{4}\right)-p_{2} k(1-q)-p_{4} q k$.

That is,

$$
f_{t_{0}+1}(k)=f_{t_{0}}(k)+p_{2} k(1-q)+p_{4} q k
$$

Since $f_{t_{0}}$ is assumed to be increasing on $[0,1]$, we must prove $p_{2}$ and $p_{4}$ are increasing functions on $[0,1]$, to guarantee that $f_{t_{0}}+1$ is an increasing function on $[0,1]$.

We first show $p_{2}$ is an increasing function on $[0,1]$. For any given public belief path $l$ leading to $h_{t_{0}}=-1$, its probability is denoted by $\hat{p}_{l}$. We denote the number of up-movement and down-movement by $m, n$, and the number of flat-movement is $t_{0}-m-n$. Then,

$$
\hat{p}_{l}=(q k)^{m}(1-q)^{n} k^{n}(1-k)^{t_{0}-m-n}=q^{m}(1-q)^{n}\left(\frac{k}{1-k}\right)^{m+n}(1-k)^{t_{0}} .
$$

Obviously, $\hat{p}_{l}$ is an increasing function of $k$ on $[0,1]$. Denoting the set of paths leading to state $i$ by $L_{t_{0}}^{i}$, we have

$$
p_{2}=\sum_{l \in L_{t_{0}}^{-1}} \hat{p}_{l}
$$

Recalling that $\hat{p}_{l}, l \in L_{t_{0}}^{-1}$, are increasing functions on $[0,1]$, we have $p_{2}$ is an increasing function on $[0,1]$. 
Following the same lines, we can prove that $p_{4}$ is an increasing function on $[0,1]$. According to (C.3), it is clear that $f_{t_{0}+1}$ is an increasing function on $[0,1]$, if $f_{t_{0}}$ is an increasing function on $[0,1]$. By the induction method, we have proved that $f_{t}$ is an increasing function on $[0,1]$, for $t \in\{2, \ldots, T\}$. The statement (i) is proved.

(ii) Assuming the nature state is $\omega=1$, we define

$$
\mathcal{E}_{t}(k):=\mathbb{P}\left(a_{t}=1 \mid k, \delta=0\right) .
$$

The statement (ii) is equivalent to the case that $\mathcal{E}_{t}$ is an increasing function on $[0,1]$ for any $t \in\{2, \ldots, T\}$.

At time $t=2$, the distribution of public belief is given by

$$
\begin{aligned}
& \bar{p}_{1}=P\left(h_{2}=-2 \mid 0<k \leq 1, \delta=0\right)=k^{2}(1-q)^{2}, \\
& \bar{p}_{2}=P\left(h_{2}=-1 \mid 0<k \leq 1, \delta=0\right)=2 k(k-1)(q-1), \\
& \bar{p}_{3}=P\left(h_{2}=0 \mid 0<k \leq 1, \delta=0\right)=(k-1)^{2}-2 k^{2} q(q-1)-2 k q(k-1), \\
& \bar{p}_{4}=P\left(h_{2}=1 \mid 0<k \leq 1, \delta=0\right)=-2 k q(k-1), \\
& \bar{p}_{5}=P\left(h_{2}=2 \mid 0<k \leq 1, \delta=0\right)=k^{2} q^{2} .
\end{aligned}
$$

The expected payoff $\mathcal{E}_{2}$ is

$$
\begin{aligned}
\mathcal{E}_{2}(k) & =q\left(\bar{p}_{2}+\bar{p}_{3}+\bar{p}_{4}\right)+\bar{p}_{5} \\
& =-2 k^{2} q^{3}+3 k^{2} q^{2}+\left(1-k^{2}\right) q .
\end{aligned}
$$

Simple calculation, omitted here, gives $\mathcal{E}_{2}$ an increasing function on $[0,1]$ for $q \in(1 / 2,1)$. Assuming $\mathcal{E}_{t_{0}}$ is an increasing function, we are going to prove $\mathcal{E}_{t_{0}+1}$ is an increasing function as well for $t_{0} \in\{2, \ldots, T\}$. Then, by the induction method, the statement (ii) will be proved.

Following the distribution defined in the proof of statement (i), we have

$$
\begin{aligned}
\mathcal{E}_{t_{0}}(k) & =q\left(p_{2}+p_{3}+p_{4}\right)+p_{5}, \\
\mathcal{E}_{t_{0}+1}(k) & =q\left(\tilde{p}_{2}+\tilde{p}_{3}+\tilde{p}_{4}\right)+\tilde{p}_{5} \\
& =q\left(p_{2}(1-(1-q) k)+p_{3}+p_{4}(1-q k)\right)+p_{4} q k+p_{5} \\
& =\mathcal{E}_{t_{0}}(k)-q p_{2}(1-q) k-q^{2} p_{4} k+p_{4} q k .
\end{aligned}
$$


Now we prove $g$ is an increasing function on $[0,1]$, where

$$
g(k)=-q p_{2}(1-q) k-q^{2} p_{4} k+p_{4} q k=q(1-q) k\left(p_{4}-p_{2}\right) .
$$

That is, we prove $\hat{f}:=p_{4}-p_{2}$ is an increasing function on $[0,1]$.

Note that the path $l_{1}$ to $h_{t_{0}}=1$ must have a symmetric path $l_{-1}$ to $h_{t_{0}}=-1$. We denote the number of up-movement and down-movement by $m, n$, and the number of flat-movement is $t_{0}-m-n$. In this setting, $m>n$, otherwise the path cannot lead to $h_{t_{0}}=1$. Accordingly, we have

$$
\begin{aligned}
\hat{f}(k) & =p_{4}-p_{2} \\
& =(g k)^{m}(1-q)^{n} k^{n}(1-k)^{t_{0}-m-n}-(g k)^{n}(1-q)^{m} k^{m}(1-k)^{t_{0}-m-n} \\
& =\left(\frac{k}{1-k}\right)^{m-n}(1-k)^{t_{0}} q^{m}(1-q)^{n}\left(1-\left(\frac{q}{1-q}\right)^{n-m}\right) .
\end{aligned}
$$

Since $m>n$ and $q \in(1 / 2,1)$, we have $\hat{f}$ is an increasing function on [0,1]. Recalling (C.4), we have $g$ is an increasing function on $[0,1]$.

Since $\mathcal{E}_{t_{0}+1}(k)=\mathcal{E}_{t_{0}}(k)+g(k), \mathcal{E}_{t_{0}+1}$ is an increasing function on $[0,1]$, if $\mathcal{E}_{t_{0}}$ is an increasing function on $[0,1]$. By the induction method, we can conclude that $\mathcal{E}_{t}$ is an increasing function on $[0,1]$ for $t \in\{2, \ldots, T\}$. The proof is completed.

\section{Proof for Proposition 2.}

Proof. According to Proposition 1, we have that

$$
\mathbb{P}\left(a_{t}=\omega \mid k=1, \delta=0, t>t_{0}\right)>\mathbb{P}\left(a_{t}=\omega \mid \delta=0,1>k>0, t>t_{0}\right) .
$$

The left hand is just the probability of up cascade (correct) in the baseline model (Bikhchandani et al., 1992), given by

$$
\frac{q_{\theta}\left(q_{\theta}+1\right)\left[1-\left(q_{\theta}-q_{\theta}^{2}\right)^{T / 2}\right]}{2\left(1-q_{\theta}+q_{\theta}^{2}\right)}<e:=\frac{q_{\theta}\left(q_{\theta}+1\right)}{2\left(1-q_{\theta}+q_{\theta}^{2}\right)} .
$$

Hence, to prove the statements of Proposition 2, it is sufficient to prove

$$
\mathbb{P}\left(a_{t}=\omega \mid 0<\delta<k, t>t_{0}\right)>e .
$$

For a given even number $T$, we denote $x$ the value of public belief at time $T$, and denote $P_{T, x}$ the probability of public belief value being $x$ at time $T$ in the selective 
disclosure model. Before information cascade, guesses disclosed in the complete disclosure setting are the same as those in the selective disclosure setting. Hence, at this stage, there is no difference in efficiency. We only prove $P_{T, x}>e$ after information cascade arises, i.e., $\left|h_{t}\right|>1$.

Without loss of generality, we assume that information cascade rises at time $T_{0}$ in the complete disclosure setting. During the period from $T_{0}$ to $T$, there are $N\left(N=T-T_{0}\right.$ ) updates of public belief in the selective disclosure setting. Denote $T_{1}$ and $T_{2}$ the UP updates $\left(h_{t}+\theta\right)$ and Down updates $\left(h_{t}-\eta\right)$ when $h_{t}>1$. Correspondingly, denote $T_{3}$ and $T_{4}$ the UP updates $\left(h_{t}+\eta\right)$ and DOWN updates $\left(h_{t}-\theta\right)$ when $h_{t}<-1$. That is,

$$
T_{1}+T_{2}+T_{3}+T_{4}=N
$$

According to the law of large numbers, we can assume that

$$
\frac{T_{1}}{T_{2}}=\frac{q_{\theta}}{1-q_{\theta}},
$$

and

$$
\frac{T_{3}}{T_{4}}=\frac{1-\lambda q_{\theta}}{\lambda q_{\theta}}
$$

As the limit case $(N \rightarrow \infty)$. When $N$ is large enough, we have

$$
T_{1}=\frac{q_{\theta}}{1-q_{\theta}} T_{2}, \quad \text { and } \quad T_{3}=\frac{1-\lambda q_{\theta}}{\lambda q_{\theta}} T_{4} .
$$

By substituting (C.6) into (C.5), we have

$$
\frac{1}{1-q_{\theta}} T_{2}+\frac{1}{\lambda q_{\theta}} T_{4}=N
$$

On the other hand,

$$
\left(T_{1}-T_{4}\right) \theta+\left(T_{3}-T_{2}\right) \eta=x
$$

The probability $P_{N, x}$ of this path on the time period $\left(T_{0}, T\right)$ is given by

$$
P_{N, x}:=q_{\theta}^{T_{1}}\left(1-q_{\theta}\right)^{T_{2}}\left(1-\lambda q_{\theta}\right)^{T_{3}}\left(\lambda q_{\theta}\right)^{T_{4}},
$$

and the probability $P_{N,-x}$ of the symmetric path is

$$
P_{N,-x}:=\left(\lambda q_{\theta}\right)^{T_{1}}\left(1-\lambda q_{\theta}\right)^{T_{2}}\left(1-q_{\theta}\right)^{T_{3}} q_{\theta}^{T_{4}} .
$$

To prove $P_{N, x}>e$ and $P_{N,-x}<1-e$, we define $f\left(\lambda, q_{\theta}\right)$ by

$$
f\left(\lambda, q_{\theta}\right):=\frac{P_{N, x}}{P_{N,-x}}=\lambda^{T_{4}-T_{1}}\left(\frac{1-q_{\theta}}{1-\lambda q_{\theta}}\right)^{T_{2}-T_{3}} .
$$


To simplify the notations, we define

$$
M_{1}=T_{1}-T_{4}, \quad \text { and } \quad M_{2}=T_{3}-T_{2} .
$$

Recalling (C.6), we have

$$
\left\{\begin{array}{l}
M_{1}=\frac{p}{1-p} T_{2}-T_{4}, \\
M_{2}=\frac{1-\lambda p}{\lambda p} T_{4}-T_{2} .
\end{array}\right.
$$

Then, $T_{2}, T_{4}$ can be expressed in terms of $M_{1}, M_{2}$ as follows

$$
\left\{\begin{array}{l}
T_{2}=\frac{\lambda\left(1-q_{\theta}\right)}{1-\lambda}\left(\frac{1-\lambda q_{\theta}}{\lambda q_{\theta}} M_{1}+M_{2}\right), \\
T_{4}=\frac{\lambda\left(1-q_{\theta}\right)}{1-\lambda}\left(M_{1}+\frac{q_{\theta}}{1-q_{\theta}} M_{2}\right) .
\end{array}\right.
$$

Substituting (C.9) into (C.5), we have

$$
M_{1} b_{1}+M_{2} b_{2}=T
$$

where $b_{1}=\frac{2-\lambda q_{\theta}-q_{\theta}}{(1-\lambda) q_{\theta}}$, and $b_{2}=\frac{1+\lambda}{1-\lambda}$.

According to (C.8) and (C.10), we have

$$
\left\{\begin{array}{l}
M_{1}=\frac{b_{1} x-N \theta}{\eta b_{1}-b_{2} \theta} \\
M_{2}=\frac{b_{2} x-N \eta}{\theta b_{2}-b_{1} \eta} .
\end{array}\right.
$$

Now we are ready to prove $P_{N, x}>e$ and $P_{N,-x}<1-e$, which is equivalent to

$$
f\left(\lambda, q_{\theta}\right)>\frac{e}{1-e} .
$$

Since $0<e<1$, inequality (C.12) is equivalent to

$$
\log \left(f\left(\lambda, q_{\theta}\right)\right)>\log (e /(1-e)),
$$

that is,

$$
-M_{1} \log \lambda-M_{2} \log \left(\frac{1-q_{\theta}}{1-\lambda q_{\theta}}\right)>\log (e /(1-e)) .
$$

Inequality (C.13)can be rewritten as

$$
-M_{1} \kappa_{1}-M_{2} \kappa_{2}>\log (e /(1-e)),
$$

where $\kappa_{1}=\log \lambda$, and $\kappa_{2}=\log \left(\frac{1-q_{\theta}}{1-\lambda q_{\theta}}\right)$. 
Recalling (C.11), we have to prove

$$
-\frac{b_{1} x-N \theta}{\eta b_{1}-b_{2} \theta} \kappa_{1}-\frac{b_{2} x-N \eta}{\theta b_{2}-b_{1} \eta} \kappa_{2}>\log (e /(1-e)) .
$$

That is,

$$
N\left(\frac{\eta \kappa_{1}-\theta \kappa_{2}}{\theta b_{2}-b_{1} \eta}\right)-x\left(\frac{b_{2} \kappa_{1}-b_{1} \kappa_{2}}{\theta b_{2}-b_{1} \eta}\right)>\log \left(\frac{e}{1-e}\right) .
$$

For given $x$ and $e$, if $\frac{\eta \kappa_{1}-\theta \kappa_{2}}{\theta b_{2}-b_{1} \eta}>0$, inequality (C.14) should hold. Thus, we must prove

$$
\left(\eta \kappa_{1}-\theta \kappa_{2}\right)\left(\theta b_{2}-b_{1} \eta\right)>0
$$

That is,

$$
-\eta^{2} \kappa_{1} b_{1}-\theta^{2} \kappa_{2} b_{2}+\left(\kappa_{1} b_{2}+\kappa_{2} b_{1}\right) \theta \eta>0
$$

Inequality (C.15) can be rewritten as

$$
\left(\begin{array}{ll}
\eta & \theta
\end{array}\right) A\left(\begin{array}{l}
\eta \\
\theta
\end{array}\right)>0
$$

where

$$
A=\left(\begin{array}{cc}
-\kappa_{1} b_{1} & \frac{\kappa_{1} b_{2}+\kappa_{2} b_{1}}{2} \\
\frac{\kappa_{1} b_{2}+\kappa_{2} b_{1}}{2} & -\kappa_{2} b_{2}
\end{array}\right) .
$$

Since $\kappa_{1} b_{1}<0$ and $|A|=\frac{1}{4}\left(\kappa_{1} b_{2}-\kappa_{2} b_{1}\right)^{2}>0, A$ is a positive-definite matrix. That is, inequality (C.15) or (C.16) holds if at most one of $\eta$ and $\theta$ is zero. Thus, we have proved that $P_{N, x}>e$ and $P_{N,-x}<1-e$. Hence, the conclusion of Proposition 2 was proved. 